\title{
Measure-Dependent Stochastic Nonlinear Beam Equations Driven by Fractional Brownian Motion
}

\author{
Mark A. McKibben \\ Department of Mathematics, West Chester University of Pennsylvania, 25 University Avenue, \\ West Chester, PA 19343, USA \\ Correspondence should be addressed to Mark A. McKibben; mmckibben@wcupa.edu
}

Received 1 October 2013; Accepted 9 November 2013

Academic Editor: Ciprian A. Tudor

Copyright (C) 2013 Mark A. McKibben. This is an open access article distributed under the Creative Commons Attribution License, which permits unrestricted use, distribution, and reproduction in any medium, provided the original work is properly cited.

We study a class of nonlinear stochastic partial differential equations arising in the mathematical modeling of the transverse motion of an extensible beam in the plane. Nonlinear forcing terms of functional-type and those dependent upon a family of probability measures are incorporated into the initial-boundary value problem (IBVP), and noise is incorporated into the mathematical description of the phenomenon via a fractional Brownian motion process. The IBVP is subsequently reformulated as an abstract second-order stochastic evolution equation driven by a fractional Brownian motion ( $\mathrm{fBm}$ ) dependent upon a family of probability measures in a real separable Hilbert space and is studied using the tools of cosine function theory, stochastic analysis, and fixed-point theory. Global existence and uniqueness results for mild solutions, continuous dependence estimates, and various approximation results are established and applied in the context of the model.

\section{Introduction}

The mathematical description of the dynamic buckling has been the subject of investigation for decades and is of interest to the engineering world. Dynamic buckling arises in various ways including vibrations, single load pulses of large amplitude, occurrence of a suddenly applied load, and flutter enhanced bending (see [1]). For the purpose of our study we restrict our attention to the dynamic buckling of a hinged extensible beam which is stretched or compressed by an axial force.

Dickey [2] initiated an investigation of the hyperbolic partial differential equation

$$
\begin{aligned}
& \frac{\partial^{2} W(z, t)}{\partial t^{2}}+ \frac{E I}{\rho} \frac{\partial^{4} W(z, t)}{\partial z^{4}} \\
&-\left(\frac{\eta}{\rho}+\frac{E A_{c}}{2 \rho L} \int_{0}^{L} \frac{\partial^{2} W(\xi, t)}{\partial \xi^{2}} d \xi\right) \frac{\partial^{2} W(z, t)}{\partial z^{2}}=0, \\
& 0 \leq z \leq L, 0<t,
\end{aligned}
$$

where $E$ is Young's modulus, $I$ is the cross-sectional moment of inertia, $\rho$ is the density, $\eta$ is an axial force, $L$ is the natural length of the beam, and $A_{c}$ is the cross-sectional area; these are all positive parameters. Here, $W(z, t)$ describes the transverse deflection of an extensible beam at point $z$ at time $t$. The nonlinear term in (1) accounts for the change in tension of the beam due to extensibility. The ends of the beam are held at a fixed distance apart and the ends are hinged; this translates to the following boundary conditions:

$$
W(0, t)=W(L, t)=\frac{\partial^{2} W(0, t)}{\partial z^{2}}=\frac{\partial^{2} W(L, t)}{\partial z^{2}}=0 .
$$

The initial conditions given by

$$
W(z, 0)=\varsigma_{0}(z), \quad \frac{\partial W(z, 0)}{\partial t}=\varsigma_{1}(z), \quad 0 \leq z \leq L
$$

describe the initial deflection and initial velocity at each point $z$ of the beam.

Fitzgibbon [3] and Ball [4] established the general existence theory for IBVP (1)-(3). Patcheu [5] subsequently 
incorporated a nonlinear friction force term into the mathematical model to account for dissipation; this was done by replacing the right side of (1) by the term $\zeta|(\partial W(z, t)) / \partial t|$, where $\zeta$ is a bounded linear operator. More recently, Balachandran and Park [6] further introduced the term $-\lambda\left(\partial^{4} W(z, t) / \partial t^{2} \partial z^{2}\right)$ into (1) to account for the fact that during vibration, the elements of the beam not only undergo translator motion but also rotate.

The above results were established for the deterministic case and did not account for environmental noise. As Kannan and Bharucha-Reid [7] points out, the variability in measurement obtained experimentally suggests that studying a stochastic version of the model is advantageous. Indeed, doing so enables us to understand the effects of noise on the behavior of the phenomenon. To this end, Mahmudov and McKibben [8] studied a stochastic version of (1) (with damping), assuming that noise was incorporated into the model via a standard one-dimensional Brownian motion process.

The purpose of the present work is to generalize those results in two ways. One of the directions for this generalization is that we broaden the class of nonlinear forcing terms incorporated into the model. We do so in two ways, one of which is to consider more general functional-type forcing terms that capture general integral terms, semilinear terms, and others under the same abstract form. For the second way, we point out that accounting for certain types of nonlinearities in the mathematical modeling of some phenomena-for instance, nonlinear waves and the dynamic buckling of a hinged extensible beamrequires that the nonlinearities depend on the probability distribution of the solution process. This notion was first studied in the finite-dimensional setting $[9,10]$, was initiated in the infinite-dimensional setting by Ahmed and Ding [11], and subsequently was studied by various authors $[12,13]$.

The second direction of the generalization is to incorporate noise into the model via a more general fractional Brownian motion process. Regarding the mathematical description of the noise term, it is often the case that the standard Brownian motion is insufficient. Indeed, it has been shown that certain processes arising in a broad array of applications-such as communication networks, self-similar protein dynamics, certain financial models, and nonlinear waves-exhibit a self-similarity property in the sense that the processes $\{x(\alpha t): 0 \leq t \leq T\}$ and $\left\{\alpha^{H} x(t): 0 \leq t \leq T\right\}$ have the same law (see [14-16]). Indeed, while the case when $H=$ $1 / 2$ generates a standard Brownian motion, concrete data from a variety of applications have exhibited other values of $H$, and it seems that this difference enters in a nonnegligible way in the modeling of this phenomena. In fact, since $B^{H}(t)$ is not a semimartingale unless $H=1 / 2$, the standard stochastic calculus involving the Itó integral cannot be used in the analysis of related stochastic evolution equations. Several authors have studied the formulation of stochastic calculus for $\mathrm{fBm}$ and differential/evolution equations driven by $\mathrm{fBm}$ in the past decade [14-18].
Specifically, we consider the following stochastic IBVP:

$$
\begin{gathered}
\partial\left(\frac{\partial W(z, t ; \omega)}{\partial t}\right)+\left[\frac{E I}{\rho} \frac{\partial^{4} W(z, t ; \omega)}{\partial z^{4}}+\alpha \frac{\partial^{3} W(z, t ; \omega)}{\partial t \partial z^{2}}\right] \partial t \\
-\left[\left(\frac{H}{\rho}+\frac{E A_{c}}{2 \rho L} \int_{0}^{L}\left|\frac{\partial W(\xi, t ; \omega)}{\partial \xi}\right|^{2} d \xi\right)\right. \\
\left.\quad \times \frac{\partial^{2} W(z, t ; \omega)}{\partial z^{2}}\right] \partial t \\
=\mathscr{F}(W(z, t ; \omega), t ; \omega) \partial t+g(z, t ; \omega) d \beta^{H}(t ; \omega)
\end{gathered}
$$

$W(0, t ; \omega)=W(L, t ; \omega)=\frac{\partial^{2} W(0, t ; \omega)}{\partial z^{2}}=\frac{\partial^{2} W(L, t ; \omega)}{\partial z^{2}}=0$,

$$
W(z, 0 ; \omega)=\varsigma_{0}(z ; \omega), \quad \frac{\partial W(z, 0 ; \omega)}{\partial t}=\varsigma_{1}(z ; \omega),
$$

where $0 \leq z \leq L, 0 \leq t \leq T, \omega \in \Omega$ (a complete probability space), $\left\{\beta^{H}(t ; \omega): 0 \leq t \leq T\right\}$ is a fractional Brownian motion, and the nonlinear forcing term $\mathscr{F}(W(z, t ; \omega), t ; \omega)$ will take on various forms including

$$
\begin{gathered}
\int_{0}^{t} b(t, s) F_{0}(s, W(z, s ; \omega)) d s+F_{1}(t, W(z, t ; \omega)) \\
\int_{0}^{T} a(t, s) F_{2}\left(z, s, W(z, s ; \omega), \int_{0}^{s} k(s, \tau, W(z, \tau ; \omega)) d \tau\right) d s \\
F_{3}(z, t, W(z, t ; \omega))+\int_{\mathscr{L}^{2}(0, L)} F_{4}(z, y, t ; \omega) \mu(z, t)(d y),
\end{gathered}
$$

where $\{\mu(z, t): 0 \leq z \leq L, 0 \leq t \leq T\}$ is a family of probability measures, and $a, k$, and $F_{i}(i=1,2,3,4)$ are appropriate mappings.

The plan of the paper is as follows. We will collect some preliminary information on cosine function theory, special function spaces, probability measures, and fractional Brownian motion in Section 2. Then we present abstract formulations of the IBVPs under consideration in Section 3. The main theoretical results together with corollaries illustrating the applicability to the IBVPs introduced above are gathered in Section 4. The proofs of the main results are provided in Section 5 , followed by an interpretation of these results for the specific nonlinear beam model in Section 6. An extensive reference list then follows.

\section{Preliminaries}

For details of this section, we refer the reader to $[4,16,17$, $19,20]$ and the references therein. Throughout this paper, $U$ and $V$ are real separable Hilbert spaces with norms $\|\cdot\|_{U}$ and $\|\cdot\|_{V}$, and inner products $\langle\cdot, \cdot\rangle_{U}$ and $\langle\cdot, \cdot\rangle_{V}$ equipped with a complete orthonormal basis $\left\{e_{j} \mid j=1,2, \ldots\right\}$. Also, $(\Omega, \mathfrak{F}, P)$ 
is a complete probability space. Henceforth, for brevity, we will suppress the dependence of random variables on $\omega$.

We make use of several different function spaces throughout this paper. $\mathfrak{B} \mathfrak{Q}(U)$ is the space of all bounded linear operators on $U$, while $\mathscr{L}^{2}(U)=\mathscr{L}^{2}((0, T) ; U)$ stands for the space of all $U$-valued random variables $y$ for which $E\|y\|_{U}^{2}<$ $\infty$. Also, $C([0, T] ; U)$ stands for the space of $\mathscr{L}^{2}$-continuous $U$-valued random variables $y:[0, T] \rightarrow U$ such that

$$
\|y\|_{C([0, T] ; U)}^{2} \equiv \sup _{0 \leq t \leq T} E\|y(t)\|_{U}^{2}<\infty
$$

The space of Hilbert Schmidt operators from $V$ into $U$ is denoted by $\mathfrak{Q}(V, U)$ and is equipped with the norm $\|\cdot\|_{\mathfrak{Q}(V, U)}$.

The remaining function spaces coincide with those used in [11]; we recall them here for convenience. First, $\mathscr{B}(U)$ stands for the Borel class on $U$ and $\wp(U)$ represents the space of all probability measures defined on $\mathscr{B}(U)$ equipped with the weak convergence topology. Define $\lambda: U \rightarrow(0, \infty)$ by $\lambda(x)=1+\|x\|_{U}, x \in U$, and consider the space

$$
\begin{aligned}
\mathscr{C}(U)= & \left\{\varphi: U \longrightarrow U \mid \varphi \text { is continuous and }\|\varphi\|_{\mathscr{C}}\right. \\
& =\sup _{x \in U} \frac{\|\varphi(x)\|_{U}}{\lambda^{2}(x)}+\sup _{x \neq y \text { in } U} \frac{\|\varphi(x)-\varphi(y)\|_{U}}{\|x-y\|_{U}} \\
& <\infty\} .
\end{aligned}
$$

For $p \geq 1$, we let

$\wp_{\lambda^{p}}^{s}(U)$

$=\{m: U \longrightarrow \mathbb{R} \mid m$ is assigned measure on $U$ such that

$$
\left.\|m\|_{\lambda^{p}}=\int_{U} \lambda^{p}(x)|m|(d x)<\infty\right\}
$$

where $m=m^{+}-m^{-}$is the Jordan decomposition of $m$ and $|m|=m^{+}+m^{-}$. Then define the space $\wp_{\lambda^{2}}(U)=\wp_{\lambda^{2}}^{s}(U) \cap$ $\wp(U)$ equipped with the metric $\rho$ given by

$$
\begin{aligned}
& \rho\left(\varsigma_{1}, \varsigma_{2}\right) \\
& =\sup \left\{\int_{U} \varphi(x)\left(\varsigma_{1}-\varsigma_{2}\right)(d x):\|\varphi\|_{\mathscr{C}} \leq 1\right\} .
\end{aligned}
$$

It is known that $\left(\wp_{\lambda^{2}}(U), \rho\right)$ is a complete metric space. The space of all continuous $\wp_{\lambda^{2}}(U)$-valued functions defined on $[0, T]$ is denoted by $\mathscr{C}_{\lambda^{2}}=\mathscr{C}_{\lambda^{2}}\left([0, T] ;\left(\wp_{\lambda^{2}}(U), \rho\right)\right)$ is complete when equipped with the metric

$$
D_{T}\left(\varsigma_{1}, \varsigma_{2}\right)=\sup _{t \in[0, T]} \rho\left(\varsigma_{1}(t), \varsigma_{2}(t)\right), \quad \forall \varsigma_{1}, \varsigma_{2} \in \mathscr{C}_{\lambda^{2}} .
$$

We recall some facts about cosine families of operators defined as follows.

Definition 1. (i) The one-parameter family $\{C(t): t \in \mathbb{R}\} \subset$ $\mathfrak{B} \mathfrak{Q}(U)$, satisfying (a) $C(0)=I$,

(b) $C(t) z$ is continuous in $t$ on $\mathbb{R}$, for all $z \in U$,

(c) $C(t+s)+C(t-s)=2 C(t) C(s)$, for all $t, s \in \mathbb{R}$, is called a strongly continuous cosine family.

(ii) The corresponding strongly continuous sine family $\{S(t): t \in \mathbb{R}\} \subset \mathfrak{B} \mathfrak{Q}(U)$ is defined by $S(t) z=\int_{0}^{t} C(s) z d s$, for all $t \in \mathbb{R}$, for all $z \in U$.

(iii) The (infinitesimal) generator $A: U \rightarrow U$ of $\{C(t)$ : $t \in \mathbb{R}\}$ is given by $A z=\left.\left(d^{2} / d t^{2}\right) C(t) z\right|_{t=0}$, for all $z \in D(A)=$ $\left\{z \in U: C(\cdot) z \in C^{2}(\mathbb{R} ; U)\right\}$.

It is known that the infinitesimal generator $A$ is a closed, densely-defined operator on $U$ (see [21]). Such a cosine and (corresponding sine) family and its generator satisfy the following properties.

Proposition 2. Suppose that $A$ is the infinitesimal generator of a cosine family of operators $\{C(t): t \in \mathbb{R}\}$ (cf. Definition 1 ). Then the following hold.

(i) There exist $M_{A} \geq 1$ and $\omega \geq 0$ such that $\|C(t)\| \leq$ $M_{A} e^{\omega|t|}$ and hence, $\|S(t)\| \leq M_{A} e^{\omega|t|}$,

(ii) $A \int_{s}^{r} S(u) z d u=[C(r)-C(s)] z$, for all $0 \leq s \leq r<\infty$,

(iii) There exists $N \geq 1$ such that $\|S(s)-S(r)\| \leq$ $N\left|\int_{s}^{r} e^{w|s|} d s\right|$, for all $0 \leq s \leq r<\infty$.

The Uniform Boundedness Principle, together with (i) above, implies that both $\{C(t): t \in[0, T]\}$ and $\{S(t): t \in$ $[0, T]\}$ are uniformly bounded by $M_{*}=M_{A} e^{\omega T}$.

Next, we make precise the definition of a $U$-valued $\mathrm{fBm}$ and related stochastic integral used in this paper. The approach we use coincides with the one formulated and analyzed in $[15,17]$. Let $\left\{\beta_{j}^{H}(t) \mid t \geq 0\right\}^{\infty}$ be a sequence of independent, one-dimensional $\mathrm{fBms}$ with Hurst parameter $H \in(1 / 2,1)$ such that for all $j=1,2, \ldots$

(i) $\beta_{j}^{H}(0)=0$,

(ii) $E\left[\beta_{j}^{H}(t)-\beta_{j}^{H}(s)\right]^{2}=|t-s|^{2 H} v_{j}$,

(iii) $E\left[\beta_{j}^{H}(1)\right]^{2}=v_{j}>0$,

(iv) $\sum_{j=1}^{\infty} v_{j}<\infty$.

In such case, $\sum_{j=1}^{\infty} E\left\|\beta_{j}^{H}(t) e_{j}\right\|_{U}^{2}=t^{2 H} \sum_{j=1}^{\infty} v_{j}<\infty$, so that the following definition is meaningful.

Definition 3. For every $t \geq 0, \beta^{H}(t)=\sum_{j=1}^{\infty} \beta_{j}^{H}(t) e_{j}$ is a $U$ valued $\mathrm{fBm}$, where the convergence is understood to be in the mean-square sense.

It has been shown in [17] that the covariance operator of $\left\{\beta^{H}(t): t \geq 0\right\}$ is a positive nuclear operator $Q$ such that

$$
\operatorname{tr} Q(t, s)=\frac{1}{2} \sum_{j=1}^{\infty} v_{j}\left[t^{2 H}+s^{2 H}-|t-s|^{2 H}\right] .
$$

We now outline the discussion leading to the definition of the stochastic integral associated with $\left\{\beta^{H}(t): t \geq 0\right\}$ for 
bounded, measurable functions. To begin, assume that $g$ : $[0, T] \rightarrow \mathfrak{L}(V, U)$ is a simple function; that is, there exists $\left\{g_{i}: i=1, \ldots, n\right\} \subseteq \mathbb{R}$ such that

$$
g(t)=g_{i}, \quad \forall t_{i-1} \leq t \leq t_{i},
$$

where $0=t_{0}<t_{1}<\cdots<t_{n-1}<t_{n}=T$ and $\max _{1 \leq i \leq n}$ $\left\|g_{i}\right\|_{\mathfrak{Q}(V, U)}=K$.

Definition 4. The $U$-valued stochastic integral $\int_{0}^{T} g(t) d \beta^{H}(t)$ is defined by

$$
\begin{aligned}
\int_{0}^{T} g(t) d \beta^{H}(t) & =\sum_{j=1}^{\infty}\left(\int_{0}^{T} g(t) d \beta_{j}^{H}(t)\right) e_{j} \\
& =\sum_{j=1}^{\infty}\left(\sum_{i=1}^{n} g_{i}\left[\beta_{j}^{H}\left(t_{i}\right)-\beta_{j}^{H}\left(t_{i-1}\right)\right]\right) e_{j} .
\end{aligned}
$$

As argued in Lemma 2.2 of [17], this integral is well-defined since

$$
E\left\|\int_{0}^{T} g(t) d \beta^{H}(t)\right\|_{U}^{2} \leq K^{2} T^{2 H} \sum_{j=1}^{\infty} v_{j}<\infty
$$

Since the set of simple functions is dense in the space of bounded, measurable $\mathfrak{Q}(V, U)$-valued functions, a standard density argument can be used to extend Definition 4 to the case of a general bounded measurable integrand.

Finally, in addition to the familiar Young, Hölder, and Minkowski inequalities, the inequality of the form $\left(\sum_{i=1}^{n} a_{i}\right)^{m} \leq n^{m-1} \sum_{i=1}^{n} a_{i}^{m}$, where $a_{i}$ is a nonnegative constant $(i=1, \ldots, n)$ and $m, n \in \mathbb{N}$, will be used to establish various estimates.

\section{Abstract Formulation}

The goal of this section is to reformulate the stochastic IBVPs introduced in Section 1 as abstract stochastic evolution equations. There will be two related abstract formulations depending on the nature of the nonlinearity. We begin with the reformulation (4)-(6) assuming that the forcing term $\mathscr{F}(W(z, t ; \omega), t ; \omega)$ is given by $(9)$.

We impose the following conditions:

(A1) $F_{3}:(0, L) \times[0, T] \times \mathbb{R} \rightarrow \mathbb{R}$ satisfies the Caratheodory conditions (i.e., measurable in $(x, t)$ and continuous in the third variable) such that

(i) there exists $\overline{M_{F_{3}}}>0$ for which $\left|F_{3}(y, t, w)\right| \leq$ $\overline{M_{F_{3}}}(1+|w|)$, for all $y \in(0, L), t \in[0, T]$, and $w \in \mathbb{R}$;

(ii) there exists $M_{F_{3}}>0$ for which $\mid F_{3}\left(y, t, w_{1}\right)-$ $F_{3}\left(y, t, w_{2}\right)\left|\leq M_{F_{3}}\right| w_{1}-w_{2} \mid$, for all $y \in(0, L)$, $t \in[0, T]$, and $w_{1}, w_{2} \in \mathbb{R}$;

(A2) $F_{4}:(0, L) \times[0, T] \times \mathscr{L}^{2}(0, L) \rightarrow \mathscr{L}^{2}(0, L)$ satisfies the Caratheodory conditions such that (i) there exists $\overline{M_{F_{4}}}>0$ for which $\left\|F_{4}(y, t, w)\right\|_{\mathscr{L}^{2}(0, L)} \leq \overline{M_{F_{4}}}\left(1+\|w\|_{\mathscr{L}^{2}(0, L)}\right)$, for all $y \in(0, L), t \in[0, T]$, and $w \in \mathscr{L}^{2}(0, L)$;

(ii) $F_{4}(y, t, \cdot): \mathscr{L}^{2}(0, L) \rightarrow \mathscr{L}^{2}(0, L)$ belongs to $\mathscr{C}_{\lambda^{2}}$, for every $y \in(0, L), t \in[0, T]$;

(A3) $g:(0, L) \times[0, T] \rightarrow \mathfrak{L}\left(\mathscr{L}^{2}(0, L), \mathscr{L}^{2}(0, L)\right)$ is a bounded measurable function;

(A4) $\mu(\cdot, t) \in \wp_{\lambda^{2}}\left(\mathscr{L}^{2}(0, L)\right)$ is the probability law of $W(\cdot, t)$, for each $t \in[0, T]$;

(A5) $\varsigma_{0}(\cdot), \varsigma_{1}(\cdot) \in \mathscr{L}^{2}\left(\Omega ; \mathscr{L}^{2}(0, L)\right)$.

We reformulate the associated IBVP by making the following identifications as in [8]; we recall the highlights of that formulation here for completeness of the discussion.

Let $U=V=\mathscr{L}^{2}(0, L)$ (note they are real separable Hilbert spaces) and identify the solution process $x:[0, T] \rightarrow U$ by

$$
x(t)(z)=W(z, t), \quad \forall z \in(0, L), t \in[0, T] .
$$

Define the operator $A: D(A) \subset U \rightarrow U$ by

$$
\begin{gathered}
A x(t, \cdot)=\frac{\partial^{4} x(t, \cdot)}{\partial z^{4}}, \\
D(A)=\left\{x \in H^{4}(0, L): x(0)=x(L)=x^{\prime \prime}(0)=x^{\prime \prime}(L)=0\right\} .
\end{gathered}
$$

It is known that $A$ is a positive, self-adjoint operator on $U$ (cf. $[21,22])$. As such, $A$ generates a strongly continuous cosine family of operators on $U$. We will express the other terms on the left side of (4) using fractional powers of $A$, as in [22]. To this end, the eigenvalues of $A$ are $\left\{\lambda_{n}=(n \pi)^{4}: n=1,2,3, \ldots\right\}$ with corresponding eigenvalues

$$
\left\{z_{n}(s)=\frac{\sqrt{2}}{L} \sin (n \pi s): n=1,2,3, \ldots, s \in[0, L]\right\} .
$$

Then $A$ has spectral representation

$$
A y=\sum_{n=1}^{\infty} \lambda_{n}\left\langle y, z_{n}\right\rangle z_{n}
$$

The following operators are well defined because the fractional powers of $A$ are positive and self-adjoint:

$$
\begin{gathered}
A^{1 / 2} y=\sum_{n=1}^{\infty} \lambda_{n}^{1 / 2}\left\langle y, z_{n}\right\rangle z_{n}=-\frac{\partial^{2} y}{\partial z^{2}} \\
A^{1 / 4} y=\sum_{n=1}^{\infty} \lambda_{n}^{1 / 4}\left\langle y, z_{n}\right\rangle z_{n}=\frac{\partial y}{\partial z}
\end{gathered}
$$

Also, observe that

$$
\begin{aligned}
\left\|A^{1 / 4} y\right\|_{U}^{2} & =\left\langle A^{1 / 4} y, A^{1 / 4} y\right\rangle \\
& =\sum_{n=1}^{\infty} \lambda_{n}^{1 / 2}\left\langle y, z_{n}\right\rangle^{2}=\int_{0}^{L}\left\|\frac{\partial y}{\partial z}(w, t)\right\|_{U}^{2} d w .
\end{aligned}
$$


It can be shown using standard computations involving the inner product and properties of fractional powers of $A$ that $\left\|A^{1 / 4} x(t)\right\|_{U}^{2}$ and $\left\|A^{1 / 2} x(t)\right\|_{U}^{2}$ are uniformly bounded on $[0, T]$.

Define the mappings $f:[0, T] \times U \times \wp_{\lambda^{2}}(U) \rightarrow U, g:$ $[0, T] \rightarrow \mathfrak{L}(V, U), x_{0}(\cdot)$, and $x_{1}(\cdot)$, and the operator $B: U \rightarrow$ $U$ as follows:

$$
\begin{aligned}
& f(t, x(t), \mu(t))(z) \\
& =F_{3}(z, t, W(z, t)) \\
& +\int_{\mathscr{L}^{2}(0, L)} F_{4}(z, y, t) \mu(z, t)(d y) \\
& -\left(\frac{H}{\rho}+\frac{E A_{c}}{2 \rho L} \int_{0}^{L}\left|\frac{\partial W(\xi, t)}{\partial \xi}\right|^{2} d \xi\right) \frac{\partial^{2} W(z, t)}{\partial z^{2}} \\
& =F_{3}(z, t, W(z, t))+\int_{\mathscr{L}^{2}(0, L)} F_{4}(z, y, t) \mu(z, t)(d y) \\
& -\left(\frac{H}{\rho}+\frac{E A_{c}}{2 \rho L}\left\|A^{1 / 4} x(t)\right\|_{U}^{2}\right) A^{1 / 2} x(t), \\
& g(t)(z)=g(z, t), \\
& B\left(x^{\prime}(t)\right)(z)=\alpha \frac{\partial^{3} W(z, t)}{\partial t \partial z^{2}}=\alpha A^{1 / 2}\left(\frac{\partial W(z, t)}{\partial t^{2}}\right), \\
& x_{0}(0)(z)=\varsigma_{0}(z), \\
& x_{1}(0)(z)=\varsigma_{1}(z),
\end{aligned}
$$

for all $z \in(0, L)$ and $t \in[0, T]$.

These identifications can be used to formulate (4)-(6), with nonlinear forcing term given by (9) as the following abstract second-order stochastic evolution equation in a real separable Hilbert space $U$ :

$$
\begin{aligned}
d x^{\prime}(t)= & \left(B x^{\prime}(t)+A x(t)+f(t, x(t), \mu(t))\right) d t \\
& +g(t) d \beta^{H}(t), \quad 0 \leq t \leq T, \\
& x(0)=x_{0}, \quad x^{\prime}(0)=x_{1},
\end{aligned}
$$

$$
\mu(t)=\text { probability distribution of } x(t)
$$

in a real separable Hilbert space $U$. (By the probability distribution of $x(t)$, we mean $\mu(t)(z)=P(\{\omega \in \Omega: x(t, \omega) \epsilon$ $z\}$ ), for each $z \in \mathscr{B}(U)$.) Here, $A: D(A) \subset U \rightarrow U$ is a linear (possibly unbounded) operator which generates a strongly continuous cosine family $\{C(t): t \geq 0\}$ on $U$ with associated sine family $\{S(t): t \geq 0\} ; f:[0, T] \times U \times \wp_{\lambda^{2}}(U) \rightarrow U$; $g:[0, T] \rightarrow \mathfrak{L}(V, U)$ is a bounded, measurable mapping; $B: U \rightarrow U$ is a bounded linear operator; $\left\{\beta^{H}(t): t \geq\right.$ $0\}$ is a $V$-valued $\mathrm{fBm}$ with Hurst parameter $H \in(1 / 2,1)$; and $x_{0}, x_{1} \in \mathscr{L}^{2}(\Omega ; U)$.

We next consider the same IBVP, with the exception that the forcing term is given by a mapping of the form (7) or (8). While all other identifications remain identical, we will describe such nonlinear forcing terms more generally as a so-called functional. Precisely, we consider the following abstract second-order functional stochastic evolution equation in a real separable Hilbert space $U$ :

$$
\begin{gathered}
d x^{\prime}(t)=\left(B x^{\prime}(t)+A x(t)+\mathscr{F}(x)(t)\right) d t \\
+g(t) d \beta^{H}(t), \quad 0 \leq t \leq T, \\
x(0)=x_{0}, \quad x^{\prime}(0)=x_{1},
\end{gathered}
$$

where $\mathscr{F}: C([0, T] ; U) \rightarrow \mathscr{L}^{2}\left(0, T ; \mathscr{L}^{2}(\Omega ; U)\right)$ is a given functional and all other identifications are the same as for (26). We impose the following conditions for (7) and (8):

(A6) $\{b(t, s): 0 \leq t \leq s \leq T\} \subset \mathfrak{B} \mathfrak{Q}(U)$;

(A7) $F_{i}:(0, L) \times[0, T] \times \mathbb{R} \rightarrow \mathbb{R}(i=0,1)$ are mappings that satisfy the Caratheodory conditions and are such that there exists $M_{F_{i}}>0$ such that

$\left|F_{i}\left(y, t, w_{1}\right)-F_{i}\left(y, t, w_{2}\right)\right| \leq M_{F_{i}}\left|w_{1}-w_{2}\right|$,

for all $y \in(0, L), t \in[0, T]$, and $w_{1}, w_{2} \in \mathbb{R}$;

(A8) $F_{2}:(0, L) \times[0, T] \times \mathbb{R} \times \mathbb{R} \rightarrow \mathbb{R}$ satisfies the Caratheodory conditions and is such that there exists $M_{\mathrm{F}_{2}}>0$ such that

$$
\begin{gathered}
\left|F_{2}\left(y, t, w_{1}, z_{1}\right)-F_{2}\left(y, t, w_{2}, z_{2}\right)\right| \\
\quad \leq M_{F_{2}}\left[\left|w_{1}-w_{2}\right|+\left|z_{1}-z_{2}\right|\right],
\end{gathered}
$$

for all $y \in(0, L), t \in[0, T]$, and $w_{1}, w_{2}, z_{1}, z_{2} \in \mathbb{R}$;

(A9) $a \in \mathscr{L}^{2}\left((0, T)^{2} ; \mathbb{R}\right)$;

(A10) $k: \Delta \times \mathbb{R} \rightarrow \mathbb{R}$, where $\Delta=\{(s, t): 0<s<t<T\}$ satisfies

$$
\left|k\left(t, s, x_{1}\right)-k\left(t, s, x_{2}\right)\right| \leq M_{k}\left|x_{1}-x_{2}\right|,
$$

for all $x_{1}, x_{2} \in \mathbb{R}$ and $(s, t) \in \Delta$. Identify the solution process $x:[0, T] \rightarrow U$ by

$$
x(t)(z)=W(z, t), \quad \forall z \in(0, L), t \in[0, T] .
$$

It can be shown that under these assumptions, the following are well-defined mappings from $C([0, T] ; U)$ into $\mathscr{L}^{2}(0$, $\left.T ; \mathscr{L}^{2}(\Omega ; U)\right)$ :

$$
\begin{aligned}
\mathscr{F}(x)(t)= & \int_{0}^{t} a(t, s) \\
& \times F_{2}\left(\cdot, s, W(\cdot, s ; \omega), \int_{0}^{s} k(s, \tau, W(\cdot, \tau ; \omega)) d \tau\right) d s,
\end{aligned}
$$

$$
\begin{aligned}
\mathscr{F} & (x)(t) \\
& =\int_{0}^{t} b(t, s) F_{0}(\cdot, s, W(\cdot, s ; \omega)) d s+F_{1}(\cdot, t, W(\cdot, t ; \omega)) .
\end{aligned}
$$

The strategy is to formulate a general theory for these two abstract second-order problems and then to apply the results to the specific IBVPs formulated in Section 1. 


\section{Statement of Results}

We consider mild solutions of (26) in the following sense.

Definition 5. A stochastic process $x \in C([0, T] ; U)$ is a mild solution of (26) if

(i)

$$
\begin{aligned}
x(t)= & S(t) x_{1}+(C(t)-S(t) B) x_{0} \\
& +\int_{0}^{t} C(t-s) B x(s) d s \\
& +\int_{0}^{t} S(t-s) f(s, x(s), \mu(s)) d s \\
& +\int_{0}^{t} S(t-s) g(s) d \beta^{H}(s), \quad 0 \leq t \leq T,
\end{aligned}
$$

(ii) $\mu(t)$ is the probability distribution of $x(t)$, for all $0 \leq$ $t \leq T$.

The following conditions on (26) are imposed on the data and mappings in (26):

(A11) $A: D(A) \subset U \rightarrow U$ is the infinitesimal generator of a strongly continuous cosine family $\{C(t): t \geq 0\}$ on $U$ with associated sine family $\{S(t): t \geq 0\}$ on $U$;

(A12) $f:[0, T] \times U \times \wp_{\lambda^{2}}(U) \rightarrow U$ satisfies the following.

(i) There exists a positive constant $M_{f}$ such that

$$
\begin{aligned}
& E\left\|f\left(t, z_{1}, \varsigma_{1}\right)-f\left(t, z_{2}, \varsigma_{2}\right)\right\|_{U}^{2} \\
& \quad \leq M_{f}\left[E\left\|z_{1}-z_{2}\right\|_{U}^{2}+\rho^{2}\left(\varsigma_{1}, \varsigma_{2}\right)\right],
\end{aligned}
$$

globally on $[0, T] \times U \times \wp_{\lambda^{2}}(U)$,

(ii) there exists a positive constant $\overline{M_{f}}$ such that

$$
E\|f(t, z, \varsigma)\|_{U}^{2} \leq \overline{M_{f}}\left[1+E\|z\|_{U}^{2}+\|\varsigma\|_{\lambda^{2}}^{2}\right] ;
$$

globally on $[0, T] \times U \times \wp_{\lambda^{2}}(U)$;

(A13) $g:[0, T] \rightarrow \mathfrak{Q}(V, U)$ is a bounded, measurable mapping;

(A14) $B: U \rightarrow U$ is a bounded linear operator;

(A15) $\left\{\beta^{H}(t): 0 \leq t \leq T\right\}$ is a $U$-valued $\mathrm{fBm}$;

(A16) $x_{0}, x_{1} \in \mathscr{L}^{2}(\Omega ; U)$ are $\left(\mathscr{F}_{0}, \mathscr{B}(U)\right)$-measurable, where $\left\{\mathscr{F}_{t}: 0 \leq t \leq T\right\}$ is the family of $\sigma$-algebras $\mathscr{F}_{t}$ generated by $\left\{\beta^{H}(s): 0 \leq s \leq t\right\}$.

Henceforth, we write

$$
\begin{aligned}
& M^{*}=\max \left(\left\{\|S(t)\|_{\mathcal{B Q}(U)}: 0 \leq t \leq T\right\}\right. \\
&\left.\cup\left\{\|C(t)\|_{\mathfrak{B Q}(U)}: 0 \leq t \leq T\right\}\right),
\end{aligned}
$$

which is finite by (A11).

The following lemma, introduced in (me and dave) and stated here without proof, is critical in establishing several estimates.
Lemma 6. Assume that $g:[0, T] \rightarrow \mathfrak{Q}(V, U)$ satisfies (A13). Then, for all $0 \leq t \leq T$,

$$
E\left\|\int_{0}^{t} S(t-s) g(s) d B^{H}(s)\right\|_{U}^{2} \leq C_{t} \sum_{j=1}^{\infty} v_{j},
$$

where $C_{t}$ is a positive constant depending on $t, M_{S}$, and the growth bound on $g$, and $\left\{v_{j}: j \in \mathbb{N}\right\}$ is defined as in the discussion leading to Definition 4.

Let $\mu \in \mathscr{C}_{\lambda^{2}}$ be fixed and define the solution map $\Phi: C([0$, $T] ; U) \rightarrow C([0, T] ; U)$ by

$$
\begin{aligned}
\Phi(x)(t)= & S(t) x_{1}+(C(t)-S(t) B) x_{0} \\
& +\int_{0}^{t} C(t-s) B x(s) d s \\
& +\int_{0}^{t} S(t-s) f(s, x(s), \mu(s)) d s \\
& +\int_{0}^{t} S(t-s) g(s) d \beta^{H}(s), \quad 0 \leq t \leq T, \\
= & \sum_{i=1}^{5} I_{i}^{x}(t), \quad 0 \leq t \leq T .
\end{aligned}
$$

The first two integrals on the right side of (39) are taken in the Bochner sense, while the third is defined in Section 2. The operator $\Phi$ satisfies the following properties.

Lemma 7. If (A11)-(A16) hold, then $\Phi$ is a well-defined $\mathscr{L}^{2}$ continuous mapping.

Our first main result is as follows.

Theorem 8. If (A11)-(A16) hold, then (26) has a unique mild solution $x$ on $[0, T]$ with corresponding probability law $\mu \epsilon$ $\mathscr{C}_{\lambda^{2}}$. Further, for every $p \geq 1$, there exists a positive constant $C_{p}$ (depending on $p, T$, and $v_{j}$ ) such that

$$
\sup _{0 \leq t \leq T} E\|x(t)\|_{U}^{2 p} \leq C_{p}\left(1+E\left\|x_{0}\right\|_{U}^{2 p}+E\left\|x_{1}\right\|_{U}^{2 p}\right) .
$$

Mild solutions of (26) depend continuously on the initial data and probability distribution of the state process in the following sense.

Proposition 9. Assume that (A11)-(A16) hold, and let $x$ and $y$ be the mild solutions of (26) (as guaranteed to exist by Theorem 8) corresponding to initial data $x_{0}, x_{1}$ and $y_{0}, y_{1}$ with respective probability distributions $\mu_{x}$ and $\mu_{y}$. Then there exists a positive constant $M^{\prime}$ such that

$$
\begin{aligned}
E\|x(t)-y(t)\|_{U}^{2} \leq M^{\prime}[ & \left\|x_{0}-y_{0}\right\|_{\mathscr{L}^{2}(\Omega ; U)}^{2} \\
& +\left\|x_{1}-y_{1}\right\|_{\mathscr{L}^{2}(\Omega ; U)}^{2} \\
& \left.+D_{T}^{2}\left(\mu_{x}, \mu_{y}\right)\right] .
\end{aligned}
$$


Next, we formulate a result in which a deterministic initial-value problem is approximated by a sequence of stochastic equations of a particular form of (26) arising frequently in applications. Specifically, consider the deterministic initial-value problem

$$
\begin{gathered}
z^{\prime \prime}(t)=A z(t)+B z^{\prime}(t)+F(t, z(t)), \quad 0 \leq t \leq T, \\
z(0)=z_{0}, \quad z^{\prime}(0)=z_{1},
\end{gathered}
$$

where $z_{0} \in D(A), z_{1} \in E=\left\{u \in U: C(\cdot) u \in C^{1}(\mathbb{R} ; U)\right\}$, $B: U \rightarrow U$ is a bounded linear operator, $A$ satisfies (A11), and $F:[0, T] \times U \rightarrow U$ satisfies the following conditions:

(A17)

(i) there exists a positive constant $M_{F}$ such that $\left\|F\left(t, z_{1}\right)-F\left(t, z_{2}\right)\right\|_{U} \leq M_{F}\left\|z_{1}-z_{2}\right\|_{U}$ globally on $[0, T] \times U$;

(ii) there exists a positive constant $\overline{M_{F}}$ such that $\|F(t, z)\|_{U} \leq \overline{M_{F}}\|z\|_{U}$ globally on $[0, T] \times U$.

It is known that under these conditions, (42) has a unique mild solution $z$ given by the representation formula

$$
\begin{aligned}
z(t)= & S(t) x_{1}+(C(t)-S(t) B) x_{0} \\
& +\int_{0}^{t} C(t-s) B x(s) d s \\
& +\int_{0}^{t} S(t-s) F(s, x(s)) d s, \quad 0 \leq t \leq T .
\end{aligned}
$$

For each $\varepsilon>0$, consider the stochastic initial-value problem

$$
\begin{aligned}
d x_{\varepsilon}(t)=\left(A_{\varepsilon} x_{\varepsilon}(t)+B x_{\varepsilon}^{\prime}(t)\right. & \\
& \left.+\int_{U} F_{1 \varepsilon}(t, z) \mu_{\varepsilon}(t)(d z)+F_{2 \varepsilon}\left(t, x_{\varepsilon}(t)\right)\right) d t \\
+ & g_{\varepsilon}(t) d \beta^{H}(t), \quad 0 \leq t \leq T, \\
& x_{\varepsilon}(0)=z_{0}, \quad x_{\varepsilon}^{\prime}(0)=z_{1},
\end{aligned}
$$$$
\mu_{\varepsilon}(t)=\text { probability distribution of } x_{\varepsilon}(t) \text {, }
$$

in $U$. Here, we assume that $z_{0} \in D\left(A_{\varepsilon}\right)=D(A)$ and $z_{1} \in E_{\varepsilon}=$ $E$, for all $\varepsilon>0$ and that $F_{i \varepsilon}:[0, T] \times U \rightarrow U(i=1,2)$ are given mappings. We impose the following conditions on the data and mappings in (44), for each $\varepsilon>0$.

(A18) $A_{\varepsilon}: D\left(A_{\varepsilon}\right)=D(A) \rightarrow D\left(A_{\varepsilon}\right)$ generates a strongly continuous cosine family $\left\{C_{\varepsilon}(t): t \geq 0\right\}$ satisfying $C_{\varepsilon}(t) \rightarrow C(t)$ strongly as $\varepsilon \rightarrow 0^{+}$, uniformly in $t \in[0, T]$. Also, the corresponding sine family
$\left\{S_{\varepsilon}(t): t \geq 0\right\}$ satisfying $S_{\varepsilon}(t) \rightarrow S(t)$ strongly as $\varepsilon \rightarrow 0^{+}$, uniformly in $t \in[0, T]$. Moreover,

$\max \left\{\left\|C_{\varepsilon}(t)\right\|_{\mathfrak{B R}^{\prime}(U)}+\left\|S_{\varepsilon}(t)\right\|_{\mathfrak{B R}_{\mathcal{R}(U)}}: 0 \leq t \leq T\right\} \leq M^{*}$,

for all $\varepsilon>0$ and $M^{*}$ is the same bound used for the cosine and sine families generated by $A$.

(A19) $B_{\varepsilon}: U \rightarrow U$ is a bounded linear operator such that $B_{\varepsilon} \rightarrow B$ in $\mathfrak{B} \mathfrak{Q}(U)$ and $\left\|B_{\varepsilon}\right\|_{\mathfrak{B Q}(U)} \leq\|B\|_{\mathfrak{B Q}(U)}$, for all $\varepsilon>0$.

(A20) $F_{2 \varepsilon}:[0, T] \times U \rightarrow U$ is Lipschitz in the second variable (with the same Lipschitz constant $M_{F}$ used for $F$ in (A17)), and $F_{2 \varepsilon}(t, u) \rightarrow F(t, u)$ as $\varepsilon \rightarrow 0^{+}$, for all $u \in U$, uniformly in $t \in[0, T]$.

(A21) $F_{1 \varepsilon}:[0, T] \times U \rightarrow U$ is a continuous mapping such that $\int_{U} F_{1 \varepsilon}(t, z) \mu_{\varepsilon}(t)(d z) \rightarrow 0$ uniformly in $t$ as $\varepsilon \rightarrow 0^{+}$.

(A22) $g_{\varepsilon}:[0, T] \rightarrow \mathfrak{L}(V, U)$ is a bounded, measurable function such that $g_{\varepsilon}(t) \rightarrow 0$ as $\varepsilon \rightarrow 0^{+}$, uniformly in $t \in[0, T]$.

Under these assumptions, the following result holds.

Theorem 10. Let $z$ and $x_{\varepsilon}$ be the mild solutions of (42) and (44) on $[0, T]$, respectively. Then there exist a positive constant $\varsigma$ and a positive function $\psi(\varepsilon)$ (which decreases to 0 as $\varepsilon \rightarrow 0^{+}$) such that $E\left\|x_{\varepsilon}(t)-z(t)\right\|_{U}^{2} \leq \psi(\varepsilon) \exp (\varsigma t)$, for all $t \in[0, T]$.

We now turn our attention to (27). By a mild solution of (27), we mean the following.

Definition 11. A stochastic process $x \in C([0, T] ; U)$ is a mild solution of (27) if

$$
\begin{aligned}
x(t)= & S(t) x_{1}+(C(t)-S(t) B) x_{0} \\
& +\int_{0}^{t} C(t-s) B x(s) d s+\int_{0}^{t} S(t-s) \mathscr{F}(x)(s) d s \\
& +\int_{0}^{t} S(t-s) g(s) d \beta^{H}(s),
\end{aligned}
$$

for all $0 \leq t \leq T$.

We assume that conditions (A11), (A13)-(A16) hold, in addition to the following condition on the functional forcing term:

$(\mathrm{A} 23) \mathscr{F}: C([0, T] ; U) \rightarrow \mathscr{L}^{2}\left(0, T ; \mathscr{L}^{2}(\Omega ; U)\right)$ is such that there exists $M_{\mathscr{F}}>0$ for which

$$
\begin{aligned}
\|\mathscr{F}(x)-\mathscr{F}(y)\|_{\mathscr{L}^{2}} \leq M_{\mathscr{F}}\|x-y\|_{\mathscr{C}}, \\
\forall x, y \in C([0, T] ; U) .
\end{aligned}
$$


Define the solution map $\Gamma: C([0, T] ; U) \rightarrow C([0, T] ; U)$ by

$\Gamma(x)(t)=S(t) x_{1}+(C(t)-S(t) B) x_{0}$

$$
\begin{aligned}
& +\int_{0}^{t} C(t-s) B x(s) d s \\
& +\int_{0}^{t} S(t-s) \mathscr{F}(x)(s) d s \\
& +\int_{0}^{t} g(s) d \beta^{H}(s), \quad 0 \leq t \leq T, \\
& =I_{1}(t)+I_{2}(t)+I_{3}(t)+I_{5}(t) \\
& +\int_{0}^{t} S(t-s) \mathscr{F}(x)(s) d s, \quad 0 \leq t \leq T .
\end{aligned}
$$

(cf. (39)). The operator $\Gamma$ satisfies the following properties.

Lemma 12. If (A11), (A13)-(A16), and (A23) hold, then $\Gamma$ is a well-defined $\mathscr{L}^{2}$-continuous mapping.

The proof of the following theorem follows almost immediately from this result.

Theorem 13. If (A11), (A13)-(A16), and (A23) hold, then (27) has a unique mild solution $x$ on $[0, T]$ provided that $M^{*} \sqrt{2 T\left(M_{\mathscr{F}}^{2}+M_{B}^{2}\right)}<1$. Further, for every $p \geq 1$, there exists a positive constant $C_{p}$ (depending on $p, T$, and $v_{j}$ ) such that

$$
\sup _{0 \leq t \leq T} E\|x(t)\|_{U}^{2 p} \leq C_{p}\left(1+E\left\|x_{0}\right\|_{U}^{2 p}+E\left\|x_{1}\right\|_{U}^{2 p}\right)
$$

Corollary 14. Assume that (A11) and (A13)-(A16) hold.

(i) If (A6) and (A7) are satisfied, then (27) where the forcing term is given by (33) has a unique mild solution on $[0, T]$ provided that

$$
\sqrt{2} M_{*}\left[2 T\left(T M_{F_{0}} M_{B}+M_{F_{1}}\right)^{2}+M_{B}^{2}\right]^{1 / 2} T^{1 / 2}<1 .
$$

Moreover, for every $p \geq 1$, estimate (49a) holds.

(ii) If (A8)-(A10) are satisfied, then (27) where the forcing term is given by (32) has a unique mild solution on $[0, T]$ provided that

$$
\sqrt{2} M_{*}\left[\left[2 T M_{F_{2}}|a|_{\mathscr{L}^{2}}\left(1+T^{3} M_{k}\right)^{1 / 2}\right]^{2}+M_{B}^{2}\right]^{1 / 2} T^{1 / 2}<1 .
$$

Moreover, for every $p \geq 1$, estimate (49a) holds.

Finally, we remark that an approximation scheme in the spirit of Theorem 10 can be established for (27) by making the natural modifications to (42) and hypotheses (A20) and (A21).

\section{Proofs of Main Results}

Proof of Lemma 7. Let $\mu \in \mathscr{C}_{\lambda^{2}}$ be fixed and consider the solution map $\Phi$ defined in (39).

One can see from the discussion in Section 2 and the properties of $x$ that for any $x \in C([0, T] ; U), \Phi(x)(t)$ is a welldefined stochastic process, for each $0 \leq t \leq T$. In order to verify the $\mathscr{L}^{2}$-continuity of $\Phi$ on $[0, T]$, let $z \in C([0, T] ; U)$ and consider $0 \leq t^{*} \leq T$ and $|h|$ sufficiently small so that all terms are well defined. Observe that

$$
E\left\|\Phi(z)\left(t^{*}+h\right)-\Phi(z)\left(t^{*}\right)\right\|_{U}^{2} \leq 5 \sum_{i=1}^{5}\left\|I_{i}\left(t^{*}+h\right)-I_{i}\left(t^{*}\right)\right\|_{U}^{2}
$$

The strong continuity of $C(t)$ and $S(t)$ implies that

$$
\sum_{i=1}^{2} E\left\|I_{i}\left(t^{*}+h\right)-I_{i}\left(t^{*}\right)\right\|_{U}^{2} \longrightarrow 0 \quad \text { as }|h| \longrightarrow 0,
$$

for each $0 \leq t \leq T$.

Using (A11) and (A14), together with Hölder's inequality and properties of cosine operators, yields

$$
\begin{aligned}
& E\left\|I_{3}\left(t^{*}+h\right)-I_{3}\left(t^{*}\right)\right\|_{U}^{2} \\
& =E \| \int_{0}^{t^{*}}\left[C\left(t^{*}+h-s\right)-C\left(t^{*}-s\right)\right] B x(s) d s \\
& \quad+\int_{t^{*}}^{t^{*}+h} C\left(t^{*}+h-s\right) B x(s) d s \|_{U}^{2} d s \\
& \leq 2 t^{*} \int_{0}^{t^{*}} E\left\|\left[C\left(t^{*}+h-s\right)-C\left(t^{*}-s\right)\right] B x(s)\right\|_{U}^{2} d s \\
& \quad+2 \int_{t^{*}}^{t^{*}+h} E\left\|C\left(t^{*}+h-s\right) B x(s)\right\|_{U}^{2} d s \\
& \leq 2 t^{*} \int_{0}^{t^{*}} E\left\|\left[C\left(t^{*}+h-s\right)-C\left(t^{*}-s\right)\right] B x(s)\right\|_{U}^{2} d s \\
& \quad+2 h\left(M^{*}\right)^{2} M_{B}^{2}\|x\|_{C}^{2} .
\end{aligned}
$$

The strong continuity of $C(t)$, along with the Lebesgue dominated convergence theorem, implies that the right side of (52) goes to 0 as $|h| \rightarrow 0$. Regarding the fourth term of (50), similar computations involving (A11) and (A12) yield the estimate

$$
\begin{aligned}
E \| I_{4}\left(t^{*}+h\right)- & I_{4}\left(t^{*}\right) \|_{U}^{2} \\
\leq 2 T \int_{0}^{t^{*}} E \| & {\left[S\left(t^{*}+h-s\right)-S\left(t^{*}-s\right)\right] } \\
& \times f(s, x(s), \mu(s)) \|_{U}^{2} d s \\
& +2 h^{2}\left(M^{*}\right)^{2} E\|f(s, x(s), \mu(s))\|_{U}^{2} .
\end{aligned}
$$


Since

$$
E\|f(s, x(s), \mu(s))\|_{U}^{2} \leq M_{f}^{2}\left[1+\|x\|_{C}^{2}+\sup _{0 \leq s \leq T}\|\mu(s)\|_{\lambda^{2}}^{2}\right]
$$

and the right side is independent of $h$, it follows immediately from (A11) that the right side of (53) goes to 0 as $|h| \rightarrow 0$.

It remains to show that $I_{3}\left(t^{*}+h\right)-I_{3}\left(t^{*}\right) \rightarrow 0$ as $|h| \rightarrow 0$. Observe that

$$
\begin{aligned}
& I_{5}\left(t^{*}+h\right)-I_{5}\left(t^{*}\right) \\
& =E \| \int_{0}^{t^{*}+h} S\left(t^{*}+h-s\right) g(s) d \beta^{H}(s) \\
& -\int_{0}^{t^{*}} S\left(t^{*}-s\right) g(s) d \beta^{H}(s) \|_{U}^{2} \\
& =E \| \sum_{j=1}^{\infty} \int_{0}^{t^{*}+h} S\left(t^{*}+h-s\right) g(s) e_{j} d \beta_{j}^{H}(s) \\
& -\sum_{j=1}^{\infty} \int_{0}^{t^{*}} S\left(t^{*}-s\right) g(s) e_{j} d \beta_{j}^{H}(s) \|_{U}^{2} \\
& =E \| \sum_{j=1}^{\infty} \int_{t^{*}}^{t^{*}+h} S\left(t^{*}+h-s\right) g(s) e_{j} d \beta_{j}^{H}(s) \\
& +\sum_{j=1}^{\infty} \int_{0}^{t^{*}}\left[S\left(t^{*}+h-s\right)-S\left(t^{*}-s\right)\right] \\
& \times g(s) e_{j} d \beta_{j}^{H}(s) \|_{U}^{2} \\
& \leq 2 E\left[\left\|\sum_{j=1}^{\infty} \int_{t^{*}}^{t^{*}+h} S\left(t^{*}+h-s\right) g(s) e_{j} d \beta_{j}^{H}(s)\right\|_{U}^{2}\right. \\
& +\| \sum_{j=1}^{\infty} \int_{0}^{t^{*}}\left[S\left(t^{*}+h-s\right)-S\left(t^{*}-s\right)\right] \\
& \left.\times g(s) e_{j} d \beta_{j}^{H}(s) \|_{U}^{2}\right]
\end{aligned}
$$

For the moment, assume that $g$ is a simple function as defined in (16). Observe that for $m \in \mathbb{N}$, arguing as in Lemma 6 of [17] yields

$$
\begin{gathered}
E\left\|\sum_{j=1}^{m} \int_{0}^{t^{*}}\left[S\left(t^{*}+h-s\right)-S\left(t^{*}-s\right)\right] g(s) e_{j} d \beta_{j}^{H}(s)\right\|_{U}^{2} \\
=E\left\|\sum_{j=1}^{m} \sum_{k=0}^{n-1} \bar{S}_{h} g_{k} e_{j}\left(\beta_{j}^{H}\left(t_{k+1}\right)-\beta_{j}^{H}\left(t_{k}\right)\right)\right\|_{U}^{2}
\end{gathered}
$$

$$
\begin{aligned}
= & E\left\langle\sum_{j=1}^{m} \sum_{k=0}^{n-1} \bar{S}_{h} g_{k} e_{j}\left(\beta_{j}^{H}\left(t_{k+1}\right)-\beta_{j}^{H}\left(t_{k}\right)\right),\right. \\
& \left.\sum_{j=1}^{m} \sum_{k=0}^{n-1} \bar{S}_{h} g_{k} e_{j}\left(\beta_{j}^{H}\left(t_{k+1}\right)-\beta_{j}^{H}\left(t_{k}\right)\right)\right\rangle_{U} \\
\leq & \sum_{j=1}^{m} \sum_{k=0}^{n-1}\left\|\bar{S}_{h}\right\|_{\mathfrak{B} \mathfrak{Q}(U)}^{2} K^{2} E \\
& \times\left(\beta_{j}^{H}\left(t_{k+1}\right)-\beta_{j}^{H}\left(t_{k}\right), \beta_{j}^{H}\left(t_{k+1}\right)-\beta_{j}^{H}\left(t_{k}\right)\right)_{\mathbb{R}} \\
\leq & \sup _{0 \leq s \leq t^{*}}\left\|\bar{S}_{h}\right\|_{\mathfrak{B} \mathfrak{Q}(U)}^{2} K^{2} E\left(\beta_{j}^{H}\right)^{2} \\
= & \sup _{0 \leq s \leq t^{*}}\left\|\bar{S}_{h}\right\|_{\mathfrak{B} \mathfrak{Q}(U)}^{2} K^{2}\left(t^{*}\right)^{2 H} \sum_{j=1}^{m} v_{j},
\end{aligned}
$$

where $\bar{S}_{h}=S\left(t^{*}-t_{k}+h\right)-S\left(t^{*}-t_{k}\right)$. Hence,

$$
\begin{aligned}
& E\left\|\sum_{j=1}^{\infty} \int_{0}^{t^{*}}\left[S\left(t^{*}+h-s\right)-S\left(t^{*}-s\right)\right] g(s) e_{j} d \beta_{j}^{H}(s)\right\|_{U}^{2} \\
& \leq \lim _{m \rightarrow \infty} E \| \sum_{j=1}^{m} \int_{0}^{t^{*}}\left[S\left(t^{*}+h-s\right)-S\left(t^{*}-s\right)\right] \\
& \quad \times g(s) e_{j} d \beta_{j}^{H}(s) \|_{U}^{2} \\
& \leq \lim _{m \rightarrow \infty} \sup _{0 \leq s \leq t^{*}}\left\|\bar{S}_{h}\right\|_{\mathcal{B} \mathfrak{Q}(U)}^{2} K^{2}\left(t^{*}\right)^{2 H} \sum_{j=1}^{m} v_{j} \\
& =\sup _{0 \leq s \leq t^{*}}\left\|\bar{S}_{h}\right\|_{\mathfrak{B} \mathfrak{L}(U)}^{2} K^{2}\left(t^{*}\right)^{2 H} \sum_{j=1}^{\infty} v_{j},
\end{aligned}
$$

and the right side of (57) goes to 0 as $|h| \rightarrow 0$. Next, observe that

$$
\begin{gathered}
E\left\|\sum_{j=1}^{\infty} \int_{t^{*}}^{t^{*}+h} S\left(t^{*}+h-s\right) g(s) e_{j} d \beta_{j}^{H}(s)\right\|_{U}^{2} \\
=E\left\|\sum_{j=1}^{\infty} \int_{0}^{h} S(u) g\left(t^{*}+h-u\right) e_{j} d \beta_{j}^{H}\left(t^{*}+h-u\right)\right\|_{U}^{2} .
\end{gathered}
$$

Using the property $E\left(\beta_{j}^{H}(s)-\beta_{j}^{H}(t)\right)^{2}=|t-s|^{2 H} v_{j}$ with $s=$ $t^{*}+h$ and $t=t^{*}$, we can argue as above to conclude that the right side of (58) goes to 0 as $|h| \rightarrow 0$. Consequently, $I_{3}\left(t^{*}+h\right)-I_{3}\left(t^{*}\right) \rightarrow 0$ as $|h| \rightarrow 0$ when $g$ is a simple function. Since the set of all such simple functions is dense in $\mathfrak{Q}(V, U)$, a standard density argument can be used to extend this conclusion to a general bounded, measurable function $g$. This establishes the $\mathscr{L}^{2}$-continuity of $\Phi$. 
Finally, we assert that $\Phi(C([0, T] ; U)) \subset C([0, T] ; U)$. Indeed, the necessary estimates can be established as above, and when used in conjunction with Lemma 6, one can readily verify that $\sup _{0 \leq t \leq T} E\|\Phi(x)(t)\|_{U}^{2}<\infty$, for any $x \in$ $C([0, T] ; U)$. Thus, we conclude that $\Phi$ is well defined, and the proof of Lemma 7 is complete.

Proof of Theorem 8. Let $\mu \in \mathscr{C}_{\lambda^{2}}$ be fixed and consider the operator as $\Phi$ defined in (39). We know that $\Phi$ is well defined and $\mathscr{L}^{2}$-continuous from Lemma 7 . We now prove that $\Phi$ has a unique fixed point in $C([0, T] ; U)$. Indeed, for any $x, y \in$ $C([0, T] ; U),(39)$ implies that

$$
\begin{aligned}
& E\|(\Phi x)(t)-(\Phi y)(t)\|_{U}^{2} \\
& \quad \leq 2 T\left(M^{*}\right)^{2}\left(M_{B}^{2}+M_{f}^{2}\right) \int_{0}^{t} E\|x(s)-y(s)\|_{U}^{2} d s \\
& \quad=\delta \int_{0}^{t} E\|x(s)-y(s)\|_{U}^{2} d s,
\end{aligned}
$$

where $\delta=2 T\left(M^{*}\right)^{2}\left(M_{B}^{2}+M_{f}^{2}\right)$. For any natural number $n$, it follows from successive iteration of (59) that

$$
\left\|\Phi^{n} x-\Phi^{n} y\right\|_{C}^{2} \leq \frac{T \delta^{n}}{n !}\|x-y\|_{C}^{2}
$$

Since $\left(T \delta^{n} / n !\right)<1$ for large enough values of $n$, we can conclude from (60) that $\Phi^{n}$ is a strict contraction on $C$ and so for a given $T>0, \Phi$ has a unique fixed point $x_{\mu} \in C$ (by the Banach contraction mapping principle), and this coincides with a mild solution of (26) as desired.

To complete the proof, we must show that $\mu$ is, in fact, the probability law of $x_{\mu}$. To this end, let $\mathscr{L}\left(x_{\mu}\right)=\left\{\mathscr{L}\left(x_{\mu}(t)\right): t \in\right.$ $[0, T]\}$ represent the probability law of $x_{\mu}$ and define the map $\Psi: \mathscr{C}_{\lambda^{2}} \rightarrow \mathscr{C}_{\lambda^{2}}$ by $\Psi(\mu)=\mathscr{L}\left(x_{\mu}\right)$. It is not difficult to see that $\mathscr{L}\left(x_{\mu}(t)\right) \in \wp_{\lambda^{2}}(U)$, for all $t \in[0, T]$ since $x_{\mu} \in C([0, T] ; U)$. In order to verify the continuity of the map $t \mapsto \mathscr{L}\left(x_{\mu}(t)\right)$, we first comment that an argument similar to the one used to establish Lemma 7 can be used to show that for sufficiently small $|h|>0$

$$
\begin{array}{r}
\lim _{h \rightarrow 0} E\left\|x_{\mu}(t+h)-x_{\mu}(t)\right\|_{U}^{2}=0, \\
\forall 0 \leq t \leq T .
\end{array}
$$

Consequently, since for all $t \in[0, T]$ and $\varphi \in \mathscr{C}_{\lambda^{2}}$, it is the case that

$$
\begin{aligned}
& \left|\int_{U} \varphi(x)\left(\mathscr{L}\left(x_{\mu}(t+h)\right)-\mathscr{L}\left(x_{\mu}(t)\right)\right)(d x)\right| \\
& \quad=\left|E\left[\varphi\left(x_{\mu}(t+h)\right)-\varphi\left(x_{\mu}(t)\right)\right]\right| \\
& \quad \leq\|\varphi\|_{\lambda^{2}} E\left\|x_{\mu}(t+h)-x_{\mu}(t)\right\|_{U^{\prime}}
\end{aligned}
$$

and hence

$$
\begin{aligned}
\rho & \left(\mathscr{L}\left(x_{\mu}(t+h)\right), \mathscr{L}\left(x_{\mu}(t)\right)\right) \\
= & \sup _{\|\varphi\|_{\lambda^{2}} \leq 1} \int_{U} \varphi(x)\left(\mathscr{L}\left(x_{\mu}(t+h)\right)-\mathscr{L}\left(x_{\mu}(t)\right)\right)(d x) \\
\text { as }|h| \longrightarrow 0 & \longrightarrow 0
\end{aligned}
$$

for any $0 \leq t \leq T$. Thus, $t \mapsto \mathscr{L}\left(x_{\mu}(t)\right)$ is a continuous map, so that $\mathscr{L}\left(x_{\mu}\right) \in \mathscr{C}_{\lambda^{2}}$, thereby showing that $\Psi$ is well defined. In order to show that $\Psi$ has a unique fixed point in $\mathscr{C}_{\lambda^{2}}$, let $\mu, \nu \in \mathscr{C}_{\lambda^{2}}$ and let $x_{\mu}, x_{\nu}$ be the corresponding mild solutions of (26). A standard computation yields

$$
\begin{aligned}
E\left\|x_{\mu}(t)-x_{v}(t)\right\|_{U}^{2} & \\
\leq & 2 T\left(M^{*}\right)^{2}\left(M_{B}^{2}+M_{f}^{2}\right) \int_{0}^{t} E\left\|x_{\mu}(s)-x_{v}(s)\right\|_{U}^{2} d s \\
& +2 T\left(M^{*}\right)^{2} M_{f}^{2} \int_{0}^{t} \rho^{2}(\mu(s), \nu(s)) d s, \quad 0 \leq t \leq T .
\end{aligned}
$$

Observe that $\rho^{2}(\mu(s), \nu(s)) \leq D_{T}^{2}(\mu, \nu)$. Thus, continuing the inequality in (64) yields

$$
\begin{aligned}
E\left\|x_{\mu}(t)-x_{\nu}(t)\right\|_{U}^{2} & \\
\leq & 2 T\left(M^{*}\right)^{2}\left(M_{B}^{2}+M_{f}^{2}\right) \int_{0}^{t} E\left\|x_{\mu}(s)-x_{\nu}(s)\right\|_{U}^{2} d s \\
& +5 T^{2}\left(M^{*}\right)^{2} M_{f}^{2} D_{T}^{2}(\mu, \nu), \quad 0 \leq t \leq T .
\end{aligned}
$$

Applying Gronwall's lemma now yields

$$
\begin{aligned}
E\left\|x_{\mu}(t)-x_{v}(t)\right\|_{U}^{2} & \\
& \leq 5 T^{2}\left(M^{*}\right)^{2} M_{f}^{2} \exp \left(2 T\left(M^{*}\right)^{2}\left(M_{B}^{2}+M_{f}^{2}\right)\right) D_{T}^{2}(\mu, \nu) \\
& =\varsigma(T) D_{T}^{2}(\mu, \nu), \quad 0 \leq t \leq T .
\end{aligned}
$$

We can choose $0 \leq \bar{T} \leq T$ to ensure $\varsigma(\bar{T})<1$, so that taking the supremum above yields

$$
\left\|x_{\mu}-x_{\nu}\right\|_{C([0, T] ; U)}^{2} \leq \varsigma(\bar{T}) D_{T}^{2}(\mu, \nu) .
$$

Since

$$
\begin{array}{r}
\rho\left(\mathscr{L}\left(x_{\mu}(t)\right), \mathscr{L}\left(x_{\nu}(t)\right)\right) \leq E\left\|x_{\mu}(t)-x_{\nu}(t)\right\|_{U^{\prime}} \\
\forall 0 \leq t \leq T,
\end{array}
$$


(which follows directly from (62) and (63)), we have

$$
\begin{aligned}
& \|\Psi(\mu)-\Psi(\nu)\|_{\lambda^{2}}^{2} \\
& \quad=D_{\bar{T}}^{2}(\Psi(\mu), \Psi(\nu)) \\
& \quad \leq \sup _{t \in[0, \bar{T}]} E\left\|x_{\mu}(t)-x_{\nu}(t)\right\|_{U}^{2} \\
& \quad=\left\|x_{\mu}-x_{\nu}\right\|_{C([0, \bar{T}] ; U)}^{2}<\varsigma(\bar{T}) D_{\bar{T}}^{2}(\mu, \nu),
\end{aligned}
$$

so that $\Psi$ is a strict contraction on $\mathscr{C}_{\lambda^{2}}\left([0, \bar{T}] ;\left(\wp_{\lambda^{2}}(U), \rho\right)\right)$. Thus, (26) has a unique mild solution on $[0, \bar{T}]$ with probability distribution $\mu \in \mathscr{C}_{\lambda^{2}}\left([0, \bar{T}] ;\left(\wp_{\lambda^{2}}(U), \rho\right)\right)$. The solution can then be extended, by continuity, to the entire interval $[0, T]$ in finitely many steps, thereby completing the proof of the existence-uniqueness portion of the theorem.

Next, we establish the boundedness of $p$ th moments of the mild solutions of (26). Let $p \geq 1$ and observe that the standard computations yield, for all $0 \leq t \leq T$,

$$
\begin{aligned}
& E\left\|x_{\mu}(t)\right\|_{U}^{2 p} \\
& \leq 5^{p}\left[E\left\|S(t) x_{1}\right\|_{U}^{2 p}+E\left\|(C(t)-S(t) B) x_{0}\right\|_{U}^{2 p}\right. \\
& +E\left\|\int_{0}^{t} C(t-s) B x(s) d s\right\|_{U}^{2 p} \\
& +E\left\|\int_{0}^{t} S(t-s) f(s, x(s), \mu(s)) d s\right\|_{U}^{2 p} \\
& \left.+E\left\|\int_{0}^{t} S(t-s) g(s) d \beta^{H}(s)\right\|_{U}^{2 p}\right] \\
& +10^{p}\left(M^{*}\right)^{2 p}\left[E\left\|x_{1}\right\|_{U}^{2 p}+E\left\|x_{0}\right\|_{U}^{2 p}\left(1+M_{B}^{2 p}\right)\right. \\
& +\left(T M_{B} \int_{0}^{t} E\left\|x_{\mu}(s)\right\|_{U}^{2}\right)^{p} \\
& +\left(T \int_{0}^{t}\left(E\left\|x_{\mu}(s)\right\|_{U}^{2}+\|\mu(s)\|_{\lambda^{2}}^{2}\right) d s\right)^{p} \\
& \left.+\left(C_{j=1}^{\infty} v_{j}\right)^{p}\right] .
\end{aligned}
$$

Applying the Jensen and Hölder inequalities enables us to continue the string of inequalities above as follows:

$$
\begin{aligned}
\leq 10^{p}\left(M^{*}\right)^{2 p} & {\left[E\left\|x_{1}\right\|_{U}^{2 p}+E\left\|x_{0}\right\|_{U}^{2 p}\left(1+M_{B}^{2 p}\right)\right.} \\
& +T^{p} M_{B}^{p} \int_{0}^{t} E\left\|x_{\mu}(s)\right\|_{U}^{2 p} d s
\end{aligned}
$$

$$
\begin{aligned}
& +C_{T}^{*} 2^{p} \int_{0}^{t}\left(E\left\|x_{\mu}(s)\right\|_{U}^{2 p}+\|\mu(s)\|_{\lambda^{2}}^{2 p}\right) d s \\
& \left.+C_{T}^{p}\left(\sum_{j=1}^{\infty} v_{j}\right)^{p}\right] \\
& \leq 10^{p}\left(M^{*}\right)^{2 p}\left[E\left\|x_{1}\right\|_{U}^{2 p}+E\left\|x_{0}\right\|_{U}^{2 p}\left(1+M_{B}^{2 p}\right)\right. \\
& \left.+C_{T}^{*} 2^{p} T\left(\sup _{0 \leq s \leq T}\|\mu(s)\|_{\lambda^{2}}^{2 p}+C_{T}^{p}\left(\sum_{j=1}^{\infty} v_{j}\right)^{p}\right)\right] \\
& +10^{p}\left(M^{*}\right)^{2 p}\left(T^{p} M_{B}^{p}+C_{T}^{*} 2^{p}\right) \int_{0}^{t} E\left\|x_{\mu}(s)\right\|_{U}^{2 p} d s .
\end{aligned}
$$

Therefore, an application of Gronwall's lemma, followed by taking the supremum over $[0, T]$, yields the desired result. This completes the proof of Theorem 8 .

Proof of Proposition 9. Computations similar to those used leading to the contractivity of the solution map in Theorem 8 can be used, along with Gronwall's lemma, to establish this result. The details are omitted.

Proof of Theorem 10. We estimate each term of the representation formula for $E\left\|x_{\varepsilon}(t)-z(t)\right\|_{U}^{2}$ separately. We begin by observing that (A18) and (A19), together, guarantee the existence of positive constants $K_{i}$ and positive functions $\alpha_{i}(\varepsilon)$ (which decrease to zero as $\left.\varepsilon \rightarrow 0^{+}\right)(i=1,2,3)$ such that, for sufficiently small $\varepsilon$,

$$
\begin{aligned}
& \left\|S_{\varepsilon}(t) B z_{0}-S(t) B z_{0}\right\|_{U} \leq K_{1} \alpha_{1}(\varepsilon), \\
& \left\|S_{\varepsilon}(t) z_{0}-S(t) z_{0}\right\|_{U} \leq K_{2} \alpha_{2}(\varepsilon), \\
& \left\|C_{\varepsilon}(t) z_{0}-C(t) z_{0}\right\|_{U} \leq K_{3} \alpha_{3}(\varepsilon),
\end{aligned}
$$

for all $0 \leq t \leq T$.

Next, we estimate

$$
E\left\|\int_{0}^{t}\left(C_{\varepsilon}(t-s) B_{\varepsilon} x_{\varepsilon}(s)-C(t-s) B z(s)\right) d s\right\|_{U}^{2} .
$$

To this end, note that

$$
\begin{gathered}
\int_{0}^{t} E\left\|C_{\varepsilon}(t-s) B_{\varepsilon} x_{\varepsilon}(s)-C_{\varepsilon}(t-s) B_{\varepsilon} z(s)\right\|_{U}^{2} d s \\
\leq\left(M^{*}\right)^{2} M_{B}^{2} \int_{0}^{t} E\left\|x_{\varepsilon}(s)-z(s)\right\|_{U}^{2} d s,
\end{gathered}
$$

and that

$$
\begin{aligned}
& \int_{0}^{t} E\left\|C_{\varepsilon}(t-s) B_{\varepsilon} z(s)-C_{\varepsilon}(t-s) B z(s)\right\|_{U}^{2} d s \\
& \quad \leq\left(M^{*}\right)^{2} \int_{0}^{t} E\left\|B_{\varepsilon} z(s)-B z(s)\right\|_{U}^{2} d s .
\end{aligned}
$$


Since (A19) guarantees that the right side of (75) goes to zero as $\varepsilon \rightarrow 0^{+}$(uniformly in $t$ ), we know that there exist $K_{4}>0$ and positive function $\alpha_{4}(\varepsilon)$ as in (72) such that, for sufficiently small $\varepsilon>0$,

$$
\int_{0}^{t} E\left\|C_{\varepsilon}(t-s) B_{\varepsilon} z(s)-C_{\varepsilon}(t-s) B z(s)\right\|_{U}^{2} d s \leq K_{4} \alpha_{4}(\varepsilon),
$$

for all $0 \leq t \leq T$. Finally, (A18) and (A19) guarantee that the right side of

$$
\begin{gathered}
\int_{0}^{t} E\left\|C_{\varepsilon}(t-s) B z(s)-C(t-s) B z(s)\right\|_{U}^{2} d s \\
\quad \leq \int_{0}^{t}\left\|C_{\varepsilon}(t-s)-C(t-s)\right\|_{U}^{2} E\|B z(s)\|_{U}^{2} d s
\end{gathered}
$$

tends to zero as $\varepsilon \rightarrow 0^{+}$, uniformly in $t$, so that again there exist $K_{5}>0$ and positive function $\alpha_{5}(\varepsilon)$ as in (72) such that, for sufficiently small $\varepsilon>0$,

$$
\int_{0}^{t} E\left\|C_{\varepsilon}(t-s) B z(s)-C(t-s) B z(s)\right\|_{U}^{2} d s \leq K_{5} \alpha_{5}(\varepsilon),
$$

for all $0 \leq t \leq T$. Thus, using (75)-(78), together with standard inequalities, in (74) yields

$$
\begin{aligned}
E\left\|\int_{0}^{t}\left(C_{\varepsilon}(t-s) B_{\varepsilon} x_{\varepsilon}(s)-C(t-s) B z(s)\right) d s\right\|_{U}^{2} \\
\leq 16 T^{1 / 2}\left[\left(M^{*}\right)^{2} M_{B}^{2} \int_{0}^{t} E\left\|x_{\varepsilon}(s)-z(s)\right\|_{U}^{2} d s\right] \\
\quad+16 T^{1 / 2}\left(K_{4} \alpha_{4}(\varepsilon)+K_{5} \alpha_{5}(\varepsilon)\right) .
\end{aligned}
$$

Next, we estimate

$$
E\left\|\int_{0}^{t}\left(S_{\varepsilon}(t-s) F_{2 \varepsilon}\left(s, x_{\varepsilon}(s)\right)-S(t-s) F(s, z(s))\right) d s\right\|_{U}^{2} .
$$

The continuity of $F$, together with (A20), enables us to infer the existence of $K_{6}>0$ and $\alpha_{6}(\varepsilon)$ (as above) such that, for sufficiently small $\varepsilon>0$,

$$
\int_{0}^{t} E\left\|\left[S_{\varepsilon}(t-s)-S(t-s)\right] F(s, z(s))\right\|_{U}^{2} d s \leq K_{6} \alpha_{6}(\varepsilon),
$$

for all $0 \leq t \leq T$. Also, observe that Young's inequality and (A20), together, imply

$$
\begin{gathered}
\int_{0}^{t} E\left\|S_{\varepsilon}(t-s)\left[F_{2 \varepsilon}\left(s, x_{\varepsilon}(s)\right)-F(s, z(s))\right]\right\|_{U}^{2} d s \\
\leq\left(M^{*}\right)^{2} \int_{0}^{t} E \| F_{2 \varepsilon}\left(s, x_{\varepsilon}(s)\right)-F_{2 \varepsilon}(s, z(s))+ \\
\quad+F_{2 \varepsilon}(s, z(s))-F(s, z(s)) \|_{U}^{2} d s \\
\leq 4\left(M^{*}\right)^{2} \int_{0}^{t}\left[M_{F}^{2} E\left\|x_{\varepsilon}(s)-z(s)\right\|_{U}^{2}\right. \\
\left.+E\left\|F_{2 \varepsilon}(s, z(s))-F(s, z(s))\right\|_{U}^{2}\right] d s .
\end{gathered}
$$

Note that (A20) guarantees the existence of $K_{7}>0$ and $\alpha_{7}(\varepsilon)$ (as above) such that, for sufficiently small $\varepsilon>0$,

$$
E\left\|F_{2 \varepsilon}(s, z(s))-F(s, z(s))\right\|_{U}^{2} \leq K_{7} \alpha_{7}(\varepsilon)
$$

for all $0 \leq t \leq T$.

So, we can continue the inequality (82) to conclude that

$$
\begin{gathered}
\int_{0}^{t} E\left\|S_{\varepsilon}(t-s)\left[F_{2 \varepsilon}\left(s, x_{\varepsilon}(s)\right)-F(s, z(s))\right]\right\|_{U}^{2} d s \\
\quad \leq 4 M_{F}^{2} \int_{0}^{t} E\left\|x_{\varepsilon}(s)-z(s)\right\|_{U}^{2} d s+4 T K_{7} \alpha_{7}(\varepsilon) .
\end{gathered}
$$

Using (82) and (84), together with the Hölder, Minkowski, and Young inequalities, yields

$$
\begin{gathered}
E\left\|\int_{0}^{t}\left(S_{\varepsilon}(t-s) F_{2 \varepsilon}\left(s, x_{\varepsilon}(s)\right)-S(t-s) F(s, z(s))\right) d s\right\|_{U}^{2} \\
\leq 4 T^{1 / 2}\left[K_{2} \alpha_{2}(\varepsilon)+4 T K_{3} \alpha_{3}(\varepsilon)\right. \\
\left.+4\left(M^{*}\right)^{2} M_{F}^{2} \int_{0}^{t} E\left\|x_{\varepsilon}(s)-z(s)\right\|_{U}^{2} d s\right] .
\end{gathered}
$$

Next, (A21) guarantees the existence of $K_{8}>0$ and $\alpha_{8}(\varepsilon)$ (as above) such that, for sufficiently small $\varepsilon>0$,

$$
E\left\|\int_{U} F_{1 \varepsilon}(s, z) \mu_{\varepsilon}(s)(d z)\right\|_{U}^{2} \leq K_{8} \alpha_{8}(\varepsilon), \quad \forall 0 \leq s \leq T .
$$

As such, we have

$$
\begin{aligned}
& E\left\|\int_{0}^{t} S_{\varepsilon}(t-s) \int_{U} F_{1 \varepsilon}(s, z) \mu_{\varepsilon}(s)(d z) d s\right\|_{U}^{2} d s \\
& \quad \leq\left(M^{*}\right)^{2} T \int_{0}^{t} E\left\|\int_{U} F_{1 \varepsilon}(s, z) \mu_{\varepsilon}(s)(d z)\right\|_{U}^{2} \\
& \quad \leq\left(M^{*}\right)^{2} T^{2} K_{8} \alpha_{8}(\varepsilon)
\end{aligned}
$$

for all $0 \leq t \leq T$.

It remains to estimate $E\left\|\int_{0}^{t} S_{\varepsilon}(t-s) g_{\varepsilon}(s) d B^{H}(s)\right\|_{U}^{2}$. Observe that (A22) implies the existence of $K_{9}>0$ and $\alpha_{9}(\varepsilon)$ (as above) such that, for sufficiently small $\varepsilon>0$,

$$
E\left\|g_{\varepsilon}(t)\right\|_{\mathfrak{Q}(V ; U)}^{2} \leq K_{9} \alpha_{9}(\varepsilon), \quad 0 \leq t \leq T
$$


First, assume that $g_{\varepsilon}$ is a simple function. Observe that for $m \in \mathbb{N}$,

$$
\begin{aligned}
E\left\|\sum_{j=1}^{m} \int_{0}^{t} S_{\varepsilon}(t-s) g_{\varepsilon}(s) e_{j} d \beta_{j}^{H}(s)\right\|_{U}^{2} \\
=E\left\|\sum_{j=1}^{m} \sum_{k=0}^{n-1} S_{\varepsilon}\left(t-t_{k}\right) g_{k} e_{j}\left(\beta_{j}^{H}\left(t_{k+1}\right)-\beta_{j}^{H}\left(t_{k}\right)\right)\right\|_{U}^{2} \\
=E\left\langle\sum_{j=1}^{m} \sum_{k=0}^{n-1} S_{\varepsilon}\left(t-t_{k}\right) g_{k} e_{j}\left(\beta_{j}^{H}\left(t_{k+1}\right)-\beta_{j}^{H}\left(t_{k}\right)\right)\right. \\
\left.\quad \sum_{j=1}^{m} \sum_{k=0}^{n-1} S_{\varepsilon}\left(t-t_{k}\right) g_{k} e_{j}\left(\beta_{j}^{H}\left(t_{k+1}\right)-\beta_{j}^{H}\left(t_{k}\right)\right)\right\rangle \\
\quad \leq \sum_{j=1}^{m} \sum_{k=0}^{n-1}\left\|S_{\varepsilon}\left(t-t_{k}\right)\right\|_{\mathfrak{B} \mathfrak{Q}(U)}^{2} K_{9} \alpha_{9}(\varepsilon) E \\
\quad \leq \sup _{0 \leq s \leq t}\left\|S_{\varepsilon}\left(t-t_{k}\right)\right\|_{\mathfrak{B} \mathfrak{Q}(U)}^{2} K_{9} \alpha_{9}(\varepsilon) t^{2 H} \sum_{j=1}^{m} v_{j} . \\
\quad \sup _{0 \leq s \leq t}\left\|S_{\varepsilon}\left(t-t_{k}\right)\right\|_{\mathcal{B} \mathfrak{Q}(U)}^{2} K_{9} \alpha_{9}(\varepsilon) E\left(\beta_{j}^{H}\right)^{2}
\end{aligned}
$$

Hence,

$$
\begin{aligned}
& E\left\|\int_{0}^{t} S_{\varepsilon}(t-s) g_{\varepsilon}(s) d \beta^{H}(s)\right\|_{U}^{2} \\
& \quad=E\left\|\sum_{j=1}^{\infty} \int_{0}^{t} S_{\varepsilon}(t-s) g_{\varepsilon}(s) e_{j} d \beta_{j}^{H}(s)\right\|_{U}^{2} \\
& \quad \leq \lim _{m \rightarrow \infty} E\left\|\sum_{j=1}^{m} \int_{0}^{t} S_{\varepsilon}(t-s) g_{\varepsilon}(s) e_{j} d \beta_{j}^{H}(s)\right\|_{U}^{2} \\
& \quad \leq \lim _{m \rightarrow \infty}\left(\sup _{0 \leq s \leq t}\left\|S_{\varepsilon}\left(t-t_{k}\right)\right\|_{\mathfrak{B} \mathcal{Q}(U)}^{2} K_{9} \alpha_{9}(\varepsilon) t^{2 H} \sum_{j=1}^{m} v_{j}\right) \\
& \quad \leq\left(M_{S}^{2} t^{2 H} \sum_{j=1}^{\infty} v_{j}\right) K_{9} \alpha_{9}(\varepsilon), \quad 0 \leq t \leq T .
\end{aligned}
$$

Since the set of all such simple functions is dense in $\mathfrak{L}(V, U)$, a standard density argument can be used to establish estimate (90) for a general bounded, measurable function $g_{\varepsilon}$.
Now, using (72)-(90), we conclude that there exist positive constants $\bar{K}_{i}(i=1, \ldots, 9)$ and positive functions $\alpha_{i}(\varepsilon)(i=1, \ldots, 9)$ such that

$$
\begin{aligned}
E\left\|x_{\varepsilon}(t)-z(t)\right\|_{U}^{2} \leq & \sum_{i=1}^{9} \overline{K_{i}} \alpha_{i}(\varepsilon)+4\left(M^{*}\right)^{2} M_{F}^{2} \\
& \times \int_{0}^{t} E\left\|x_{\varepsilon}(s)-z(s)\right\|_{U}^{2} d s, \quad 0 \leq t \leq T,
\end{aligned}
$$

so that an application of Gronwall's lemma implies

$$
E\left\|x_{\varepsilon}(t)-z(t)\right\|_{U}^{2} \leq \Psi(\varepsilon) \exp (\varsigma t), \quad 0 \leq t \leq T,
$$

where $\varsigma=4\left(M^{*}\right)^{2} M_{F}^{2}$ and $\Psi(\varepsilon)=\sum_{i=1}^{9} \overline{K_{i}} \alpha_{i}(\varepsilon)$. This completes the proof.

Proof of Lemma 12. Using the discussion in Section 2 and the properties of $x$, it follows that for any $x \in C([0, T] ; U), \Gamma(x)(t)$ is a well-defined stochastic process, for each $0 \leq t \leq T$. In order to verify the $\mathscr{L}^{2}$-continuity of $\Gamma$ on $[0, T]$, let $z \in$ $C([0, T] ; U)$ and consider $0 \leq t^{*} \leq T$ and $|h|$ sufficiently small. Observe that

$$
\begin{aligned}
& E\left\|\Gamma(z)\left(t^{*}+h\right)-\Gamma(z)\left(t^{*}\right)\right\|_{U}^{2} \\
& \leq 5 \sum_{i=1, i \neq 4}^{5} E\left\|I_{i}\left(t^{*}+h\right)-I_{i}\left(t^{*}\right)\right\|_{U}^{2} \\
& \quad+E \| \int_{0}^{t^{*}+h} S\left(t^{*}+h-s\right) \mathscr{F}(x)(s) d s
\end{aligned}
$$

$$
-\int_{0}^{t^{*}} S\left(t^{*}-s\right) \mathscr{F}(x)(s) d s \|_{U}^{2} .
$$

Using Hölder's inequality with (A23) yields

$$
\begin{aligned}
E\left\|\int_{t^{*}}^{t^{*}+h} S\left(t^{*}+h-s\right) \mathscr{F}(x)(s) d s\right\|_{U}^{2} \\
\leq 2 M_{F}^{2}\left(M^{*}\right)^{2} h^{2} \\
\quad \times\left[1+\|x\|_{C([0, T] ; U)}^{2}+\|\mathscr{F}(0)\|_{C([0, T] ; U)}^{2}\right],
\end{aligned}
$$

which goes to 0 as $|h| \rightarrow 0$. Also,

$$
\begin{gathered}
E\left\|\int_{0}^{t^{*}}[S(h)-I] S\left(t^{*}-s\right) \mathscr{F}(x)(s) d s\right\|_{U}^{2} \\
\leq T \int_{0}^{t^{*}}\left\|[S(h)-I] S\left(t^{*}-s\right)\right\|_{\mathfrak{B} \mathfrak{Q}(U)}^{2} \\
\quad \times E\|\mathscr{F}(x)(s)\|_{U}^{2} d s \\
\leq T \sup _{0 \leq s \leq T}\left\|[S(h)-I] S\left(t^{*}-s\right)\right\|_{\mathfrak{B} \mathfrak{Q}(U)}^{2}\|\mathscr{F}(x)\|_{\mathscr{L}^{2}},
\end{gathered}
$$


and the right side goes to 0 as $|h| \rightarrow 0$ here as well. Successive applications of Hölder's inequality yields

$$
\begin{aligned}
& {\left[E\left\|\int_{0}^{t} S(t-s) \mathscr{F}(x)(s) d s\right\|_{U}^{2}\right]^{1 / 2}} \\
& \quad \leq T^{1 / 2} M^{*}\left[\int_{0}^{T}\|\mathscr{F}(x)(s)\|_{\mathscr{L}^{2}(\Omega ; U)}^{2} d s\right]^{1 / 2} \\
& \quad \leq T^{1 / 2} M^{*}\|\mathscr{F}(x)\|_{\mathscr{L}^{2}} .
\end{aligned}
$$

Subsequently, an application of (A23), together with Minkowski's inequality, enables us to continue the string of inequalities in (96) to conclude that

$$
\begin{aligned}
& {\left[E\left\|\int_{0}^{t} S(t-s) \mathscr{F}(x)(s) d s\right\|_{U}^{2}\right]^{1 / 2}} \\
& \quad \leq T^{1 / 2} M^{*}\left[M_{\mathscr{F}}\|x\|_{\mathscr{C}}+\|F(0)\|_{\mathscr{L}^{2}}\right] .
\end{aligned}
$$

Taking the supremum over $[0, T]$ in (97) then implies that $\int_{0}^{t} S(t-s) \mathscr{F}(x)(s) d s \in C([0, T] ; U)$, for any $x \in C([0, T] ; U)$.

The other estimates can be established as before, and when used in conjunction with Lemma 6, they can be used to verify that $\sup _{0 \leq t \leq T} E\|\Gamma(x)(t)\|_{U}^{2}<\infty$, for any $x \in C([0, T]$; $U)$. Thus, we conclude that $\Gamma$ is well defined, and the proof of Lemma 12 is complete.

Proof of Theorem 13. We know that $\Gamma$ is well defined and $\mathscr{L}^{2}$ continuous from Lemma 12. We now prove that $\Gamma$ has a unique fixed point in $C([0, T] ; U)$. For any $x, y \in C([0, T ; U])$

$$
\begin{aligned}
& (\Gamma x)(t)-(\Gamma y)(t) \\
& =\int_{0}^{t} C(t-s) B(x(s)-y(s)) d s \\
& \quad+\int_{0}^{t} S(t-s)[\mathscr{F}(x)(s)-\mathscr{F}(y)(s)] d s .
\end{aligned}
$$

Squaring both sides, taking the expectation in (98), and applying Young's inequality yields

$$
\begin{aligned}
& E\|(\Gamma x)(t)-(\Gamma y)(t)\|_{U}^{2} \\
& \leq 2 E\left\|\int_{0}^{t} S(t-s)[\mathscr{F}(x)(s)-\mathscr{F}(y)(s)] d s\right\|_{U}^{2} \\
& \quad+2 E\left\|\int_{0}^{t} C(t-s) B(x(s)-y(s)) d s\right\|_{U}^{2} \\
& \leq 2\left(M^{*}\right)^{2} T\left[\|\mathscr{F}(x)-\mathscr{F}(y)\|_{\mathscr{L}^{2}}^{2}+M_{B}^{2}\|x-y\|_{C}^{2}\right] \\
& \leq 2\left(M^{*}\right)^{2} T\left(M_{\mathscr{F}}^{2}+M_{B}^{2}\right)\|x-y\|_{C^{\prime}}^{2}
\end{aligned}
$$

and so

$$
\|\Gamma x-\Gamma y\|_{C}^{2} \leq 2\left(M^{*}\right)^{2} T\left(M_{\mathscr{F}}^{2}+M_{B}^{2}\right)\|x-y\|_{C}^{2} .
$$

Hence, $\Phi$ is a strict contraction, provided that $M^{*} \sqrt{2 T\left(M_{\mathscr{F}}^{2}+M_{B}^{2}\right)}<1$ and so has a unique fixed point which coincides with a mild solution of (27). This completes the proof.

Proof of Corollary 14. For (i), define $\mathscr{F}: C([0, T] ; U) \rightarrow$ $\mathscr{L}^{2}\left(0, T ; \mathscr{L}^{2}(\Omega ; U)\right)$ by

$$
\mathscr{F}(x)(t)=\int_{0}^{t} b(t, s) F_{0}(s, x(s)) d s+F_{1}(t, x(t))
$$

$$
\forall 0 \leq t \leq T
$$

The Uniform Boundedness Principle with (A6) guarantees the existence of a positive constant $M_{b}$ such that $\|B(t, s)\|_{\mathfrak{B} \mathbb{I}} \leq$ $M_{b}$, for all $0 \leq t \leq s \leq T$. Standard computations involving properties of expectation and Hölder's inequality imply that for all $x, y \in C([0, T] ; U)$,

$$
\begin{aligned}
&\|\mathscr{F}(x)-\mathscr{F}(y)\|_{\mathscr{L}^{2}}^{2} \\
& \leq 2 \int_{0}^{T}[ T M_{b}^{2} \int_{0}^{s} E\left\|F_{0}(\tau, x(\tau))-F_{0}(\tau, y(\tau))\right\|_{U}^{2} d \tau \\
&\left.+E\left\|F_{1}(s, x(s))-F_{1}(s, y(s))\right\|_{U}^{2}\right] \\
& \leq 2 T\left[T M_{b} M_{F_{0}}+M_{F_{1}}\right]\|x-y\|_{C^{*}}
\end{aligned}
$$

Thus, if we let $M_{\mathscr{F}}=2 T\left[T M_{b} M_{F_{0}}+M_{F_{1}}\right]$ in (A23) we can conclude from Theorem 13 that the IBVP has a unique mild solution on $[0, T]$, provided that (49a) holds.

For (ii), using similar reasoning with

$$
M_{\mathscr{F}}=2 M_{F_{2}} T|a|_{\mathscr{L}^{2}\left((0, T)^{2}\right)}\left(1+M_{k} T^{3}\right)^{1 / 2}
$$

enables us to invoke Theorem 13 to conclude that the IBVP has a unique mild solution on $[0, T]$, provided that (49b) holds.

\section{Application of Theory to Nonlinear Beam Model}

We now apply the results proved in Section 5 to the original nonlinear beam model. Consider the IBVP (4)-(6) equipped with the nonlinear forcing term (9).

Proposition 15. Assume (A1)-(A5). Then this IBVP has a unique mild solution $W \in C\left([0, T] ; \mathscr{L}^{2}(0, T)\right)$ with probability distribution $\mu$. Moreover, for every $p \geq 1$, there exists a constant $C_{p}$ for which

$$
\begin{aligned}
& \sup _{0 \leq t \leq T} E\|W(\cdot, t)\|_{\mathscr{L}^{2}(0, L)}^{2 p} \\
& \quad \leq C_{p}\left(1+E\left\|\xi_{0}\right\|_{\mathscr{L}^{2}(0, L)}^{2 p}+E\left\|\xi_{1}\right\|_{\mathscr{L}^{2}(0, L)}^{2 p}\right) .
\end{aligned}
$$


Proof. We have illustrated in Section 3 that the IBVP (4)-(6) equipped with (9) can be reformulated as the abstract evolution equation (26). We verify that with those identifications, (A11)-(A16) are satisfied.

We have already addressed (A11) in Section 3. To see that (A12) is satisfied, observe that (A1)(i) implies that

$$
\begin{aligned}
\left\|F_{3}(t, \cdot, x(t, \cdot))\right\|_{\mathscr{L}^{2}(0, L)} & \leq \bar{M}_{F_{3}}\left[\int_{\mathscr{D}}[1+|x(t, z)|]^{2} d z\right]^{1 / 2} \\
& \leq 2 \bar{M}_{F_{3}}\left[L+\|x(t, \cdot)\|_{\mathscr{L}^{2}(0, L)}^{2}\right]^{1 / 2} \\
& \leq 2 \bar{M}_{F_{3}}\left[\sqrt{L}+\|x\|_{C\left([0, T] ; \mathscr{L}^{2}(0, L)\right)}\right] \\
& \leq M_{F_{3}}^{*}\left[1+\|x\|_{C\left([0, T] ; \mathscr{L}^{2}(0, L)\right)}\right]
\end{aligned}
$$

for all $0 \leq t \leq T$ and $x \in C\left([0, T] ; \mathscr{L}^{2}(0, L)\right)$, where

$$
M_{F_{3}}^{*}= \begin{cases}2 \bar{M}_{F_{3}} \sqrt{L}, & \text { if } L>1, \\ 2 \bar{M}_{F_{3}}, & \text { if } L \leq 1 .\end{cases}
$$

Also, from (A1)(ii), we obtain

$$
\begin{aligned}
& \left\|F_{3}(t, \cdot, x(t, \cdot))-F_{3}(t, \cdot, y(t, \cdot))\right\|_{\mathscr{L}^{2}(0, L)} \\
& \quad \leq M_{F_{3}}\left[\int_{0}^{L}|x(t, z)-y(t, z)|^{2} d z\right]^{1 / 2} \\
& \quad \leq M_{F_{3}}\|x-y\|_{C\left([0, T] ; \mathscr{L}^{2}(0, L)\right)} .
\end{aligned}
$$

Next, using (A2)(i) together with the Hölder inequality, we observe that

$$
\begin{aligned}
& \left\|\int_{\mathscr{L}^{2}(0, L)} F_{4}(t, \cdot, y) \mu(t, \cdot)(d y)\right\|_{\mathscr{L}^{2}(0, L)} \\
& \quad=\left[\int_{0}^{L}\left[\int_{\mathscr{L}^{2}(0, L)} F_{4}(t, z, y) \mu(t, z)(d y)\right]^{2} d z\right]^{1 / 2} \\
& \quad \leq\left[\int_{0}^{L} \int_{\mathscr{L}^{2}(0, L)}\left\|F_{4}(t, z, y)\right\|_{\mathscr{L}^{2}(0, L)}^{2} \mu(t, z)(d y) d z\right]^{1 / 2} \\
& \quad \leq \bar{M}_{F_{4}}\left[\int_{0}^{L}\left(\int_{\mathscr{L}^{2}(0, L)}\left(1+\|y\|_{\mathscr{L}^{2}(0, L)}\right)^{2} \mu(t, z)(d y)\right) d z\right]^{1 / 2} \\
& \quad \leq \bar{M}_{F_{4}} \sqrt{L} \sqrt{\|\mu(t)\|_{\lambda^{2}}} \\
& \quad \leq \bar{M}_{F_{4}} \sqrt{L}\left(1+\|\mu(t)\|_{\lambda^{2}}\right), \quad \forall 0 \leq t \leq T, \mu \in \wp_{\lambda^{2}}(U) .
\end{aligned}
$$

Also, invoking (A2)(ii) enables us to see that for all $\mu, v \in$ $\wp_{\lambda^{2}}(U)$,

$$
\begin{aligned}
& \| \int_{\mathscr{L}^{2}(0, L)} F_{4}(t, \cdot, y) \mu(t, \cdot)(d y) \\
& \quad-\int_{\mathscr{L}^{2}(0, L)} F_{4}(t, \cdot, y) \nu(t, \cdot)(d y) \|_{\mathscr{L}^{2}(0, L)} \\
& \quad=\left\|\int_{\mathscr{L}^{2}(0, L)} F_{4}(t, \cdot, y)(\mu(t, \cdot)-v(t, \cdot))(d y)\right\|_{\mathscr{L}^{2}(0, L)} \\
& \quad \leq\|\rho(\mu(t), v(t))\|_{\mathscr{L}^{2}(0, L)} \\
& \quad \leq \sqrt{L} \rho(\mu(t), v(t)), \quad \forall 0 \leq t \leq T .
\end{aligned}
$$

Combining (105) and (108), we see that $f$ satisfies (A12)(i) with

$$
\bar{M}_{f_{1}}=2 \cdot \max \left\{\bar{M}_{F_{4}} \sqrt{L}, M_{F_{3}}^{*}\right\}
$$

and combining (107) and (109), we see that $f$ satisfies (A12)(ii) with

$$
M_{f}=\max \left\{M_{F_{3}}, \sqrt{L}\right\} .
$$

This verifies (A12). Next, (A13), (A15), and (A16) hold by assumption, and (A14) follows because of the uniform boundedness of $\left\{\left\|A^{1 / 2} x(t)\right\|_{\mathscr{L}^{2}(0, L)}: 0 \leq t \leq T\right\}$. Thus, we can invoke Theorem 8 to conclude that this IBVP has a unique mild solution $x \in C\left([0, T] ; \mathscr{L}^{2}(0, L)\right)$ with probability law $\{\mu(t, \cdot): 0 \leq t \leq T\}$.

Similar results can be established for the IBVP (4)-(6) equipped with the nonlinear forcing term described by (32) or (33) by using Corollary 14 and Theorem 13. We omit the details.

Finally, we remark that the mild solutions for each of the IBVPs introduced in Section 1 depend continuously on the initial data and that the approximation scheme in which the impact of the stochastic term can be made sufficiently negligible (cf. Theorem 10) can be applied to each of these IBVPs.

\section{References}

[1] H. E. Lindberg, Little Book of Dynamic Buckling, LCE Science/Software, 2003.

[2] R. W. Dickey, "Free vibrations and dynamic buckling of the extensible beam," Journal of Mathematical Analysis and Applications, vol. 29, no. 2, pp. 443-454, 1970.

[3] W. E. Fitzgibbon, "Global existence and boundedess of solutions to the extensible beam equation," SIAM Journal on Mathematical Analysis, vol. 13, pp. 739-745, 1982.

[4] J. M. Ball, "Initial-boundary value problems for an extensible beam," Journal of Mathematical Analysis and Applications, vol. 42, no. 1, pp. 61-90, 1973.

[5] S. K. Patcheu, "On a global solution and asymptotic behaviour for the generalized damped extensible beam equation," Journal of Differential Equations, vol. 135, no. 2, pp. 299-314, 1997. 
[6] K. Balachandran and J. Y. Park, "Existence of solutions of a class of abstract second order nonlinear integrodifferential equations," Journal of Applied Mathematics and Stochastic Analysis, vol. 15, no. 2, pp. 115-124, 2002.

[7] D. Kannan and A. T. Bharucha-Reid, "On a stochastic integrodifferential evolution equation of Volterra type," Journal of Integral Equations and Applications, vol. 10, pp. 351-379, 1985.

[8] N. I. Mahmudov and M. A. McKibben, "Abstract secondorder damped McKean-Vlasov stochastic evolution equations," Stochastic Analysis and Applications, vol. 24, no. 2, pp. 303-328, 2006.

[9] P. J. Chen and M. E. Curtin, "On a theory of heat conduction involving two temperatures," Zeitschrift für Angewandte Mathematik und Physik, vol. 19, pp. 614-627, 1968.

[10] D. A. Dawson and J. Gärtner, "Large deviations for the McKeanVlasov limit for weakly interacting diffusions," Stochastics, vol. 20, pp. 247-308, 1987.

[11] N. U. Ahmed and X. Ding, "A semilinear Mckean-Vlasov stochastic evolution equation in Hilbert space," Stochastic Processes and their Applications, vol. 60, no. 1, pp. 65-85, 1995.

[12] D. N. Keck and M. A. McKibben, "Functional integro-differential stochastic evolution equations in Hilbert space," Journal of Applied Mathematics and Stochastic Analysis, vol. 16, no. 2, pp. 141-161, 2003.

[13] M. McKibben, "Second order damped functional stochastic evolution equations in Hilbert spaces," Dynamic Systems and Applications, vol. 12, no. 3-4, pp. 467-488, 2003.

[14] L. Decreusefond and A. S. Üstünel, "Stochastic analysis of the fractional brownian motion," Potential Analysis, vol. 10, no. 2, pp. 177-214, 1999.

[15] W. Grecksch and V. V. Anh, "A parabolic stochastic differential equation with fractional Brownian motion input," Statistics and Probability Letters, vol. 41, no. 2, pp. 337-346, 1999.

[16] B. Maslowski and D. Nualart, "Evolution equations driven by a fractional Brownian motion," Journal of Functional Analysis, vol. 202, no. 1, pp. 277-305, 2003.

[17] V. V. Anh and W. Greksch, "A fractional stochastic evolution equation driven by fractional Brownian motion," Monte Carlo Methods and Applications, vol. 9, no. 3, pp. 189-199, 2003.

[18] T. E. Duncan, B. Pasik-Duncan, and B. Maslowski, "Fractional brownian motion and stochastic equations in hilbert space," Stochastics and Dynamics, vol. 2, no. 2, pp. 225-250, 2002.

[19] J. Bochenek, "An abstract nonlinear second-order differential equation," Annales Polonici Mathematici, vol. 2, pp. 155-166, 1991.

[20] J. P. Dauer and N. Mahmudov, "Remark on existence result for second order evolution equations in Banach spaces," International Journal of Pure and Applied Mathematics, vol. 12, no. 4, pp. 471-482, 2004.

[21] H. O. Fattorini, Second Order Linear Differential Equations in Banach Spaces, vol. 108 of North Holland Mathematics Studies, Elsevier Science, Amsterdam, The Netherlands, 1985.

[22] A. Pazy, Semigroups of Linear Operators and Applications to Partial Differential Equations, Springer, New York, NY, USA, 1983. 


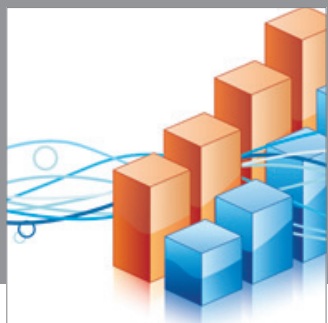

Advances in

Operations Research

mansans

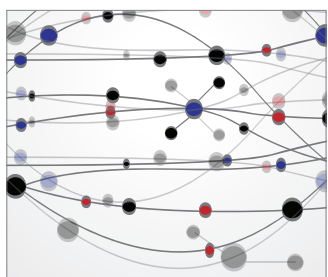

The Scientific World Journal
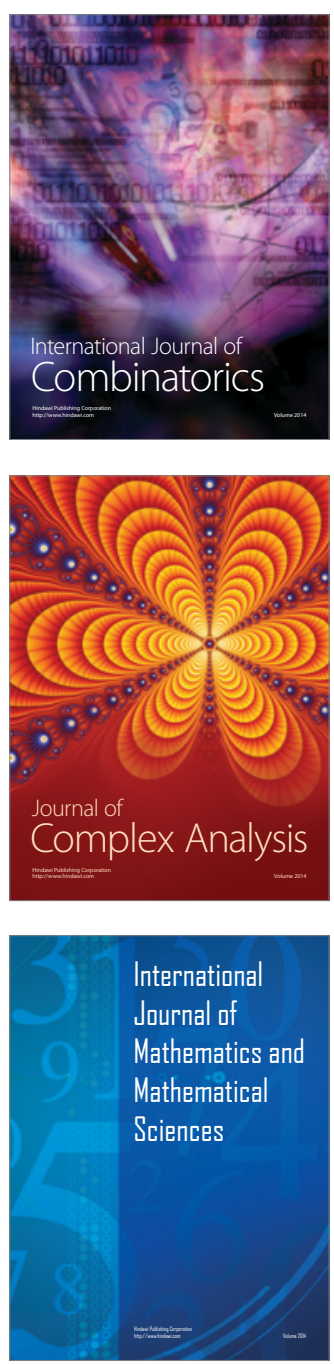
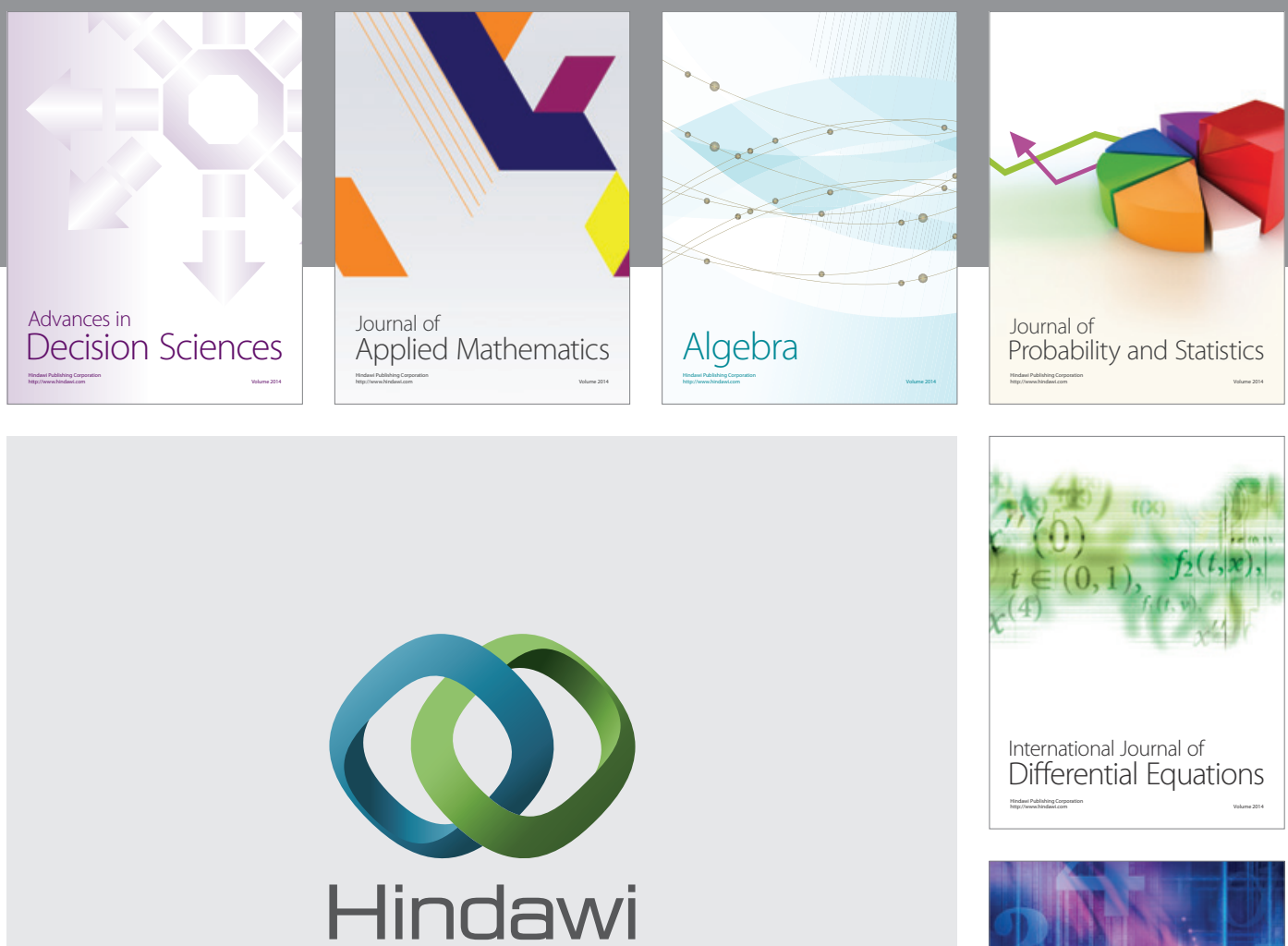

Submit your manuscripts at http://www.hindawi.com
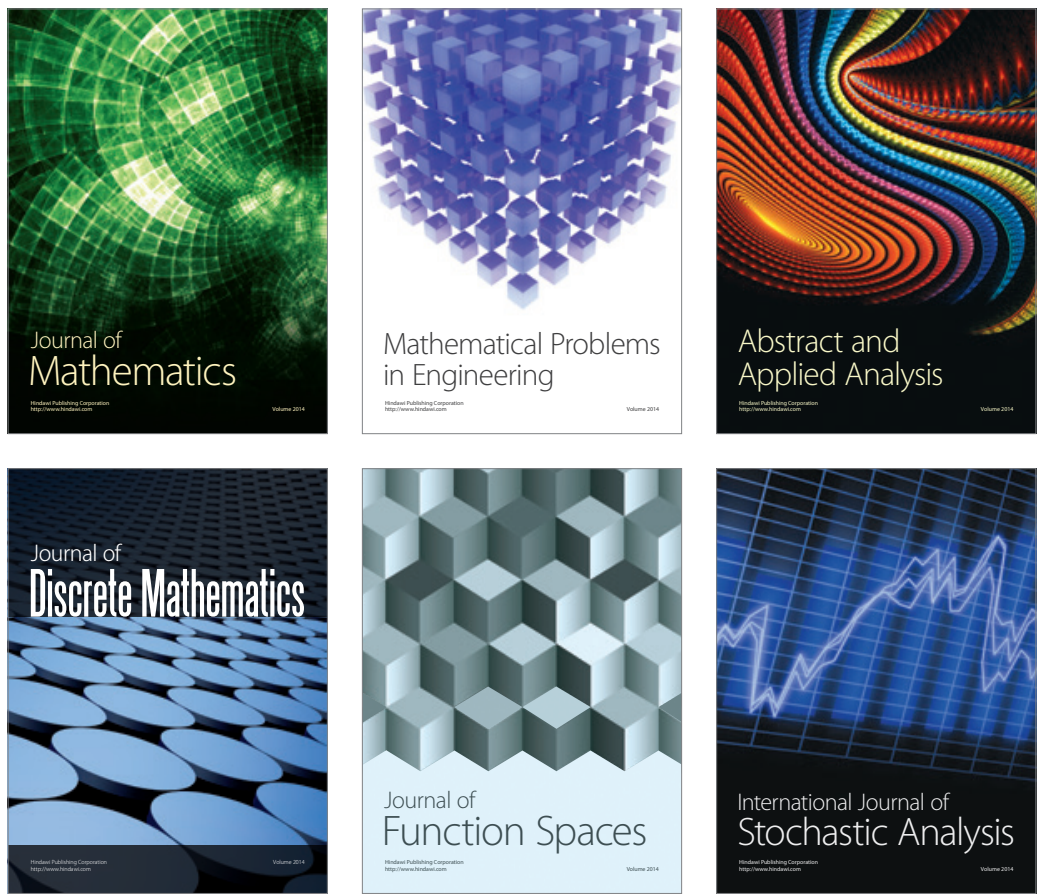

Journal of

Function Spaces

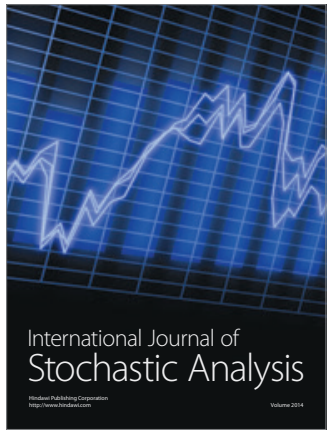

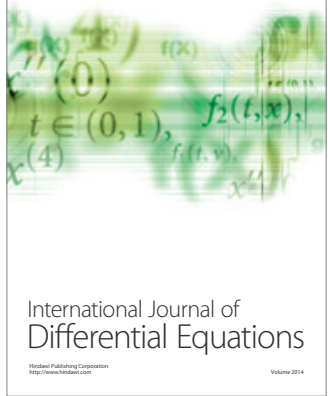
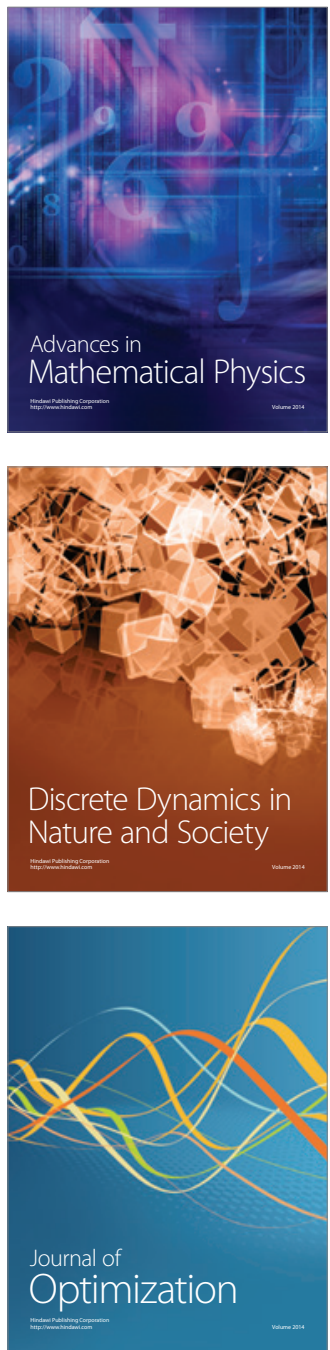